\title{
The combination of GNSS-levelling data and gravimetric (quasi-) geoid heights in the presence of noise
}

\author{
R. Klees · I. Prutkin
}

Received: 22 January 2010 / Accepted: 12 August 2010 / Published online: 29 August 2010

(C) The Author(s) 2010. This article is published with open access at Springerlink.com

\begin{abstract}
We propose a methodology for the combination of a gravimetric (quasi-) geoid with GNSS-levelling data in the presence of noise with correlations and/or spatially varying noise variances. It comprises two steps: first, a gravimetric (quasi-) geoid is computed using the available gravity data, which, in a second step, is improved using ellipsoidal heights at benchmarks provided by GNSS once they have become available. The methodology is an alternative to the integrated processing of all available data using least-squares techniques or least-squares collocation. Unlike the correctorsurface approach, the pursued approach guarantees that the corrections applied to the gravimetric (quasi-) geoid are consistent with the gravity anomaly data set. The methodology is applied to a data set comprising 109 gravimetric quasi-geoid heights, ellipsoidal heights and normal heights at benchmarks in Switzerland. Each data set is complemented by a full noise covariance matrix. We show that when neglecting noise correlations and/or spatially varying noise variances, errors up to $10 \%$ of the differences between geometric and gravimetric quasi-geoid heights are introduced. This suggests that if highquality ellipsoidal heights at benchmarks are available and are used to compute an improved (quasi-) geoid, noise covariance matrices referring to the same datum should be used in the data processing whenever they are available. We compare the methodology with the corrector-surface approach using various corrector surface models. We show that the commonly used corrector surfaces fail to model the more complicated spatial patterns of differences between geometric and gravimetric quasi-geoid heights present in the data set. More flexible parametric models such as radial basis
\end{abstract}

R. Klees $(\varangle) \cdot$ I. Prutkin

Delft Institute of Earth Observation and Space Systems (DEOS), Delft University of Technology, Kluyverweg 1,

2629 HS Delft, The Netherlands

e-mail: r.klees@tudelft.nl function approximations or minimum-curvature harmonic splines perform better. We also compare the proposed method with generalized least-squares collocation, which comprises a deterministic trend model, a random signal component and a random correlated noise component. Trend model parameters and signal covariance function parameters are estimated iteratively from the data using non-linear least-squares techniques. We show that the performance of generalized least-squares collocation is better than the performance of corrector surfaces, but the differences with respect to the proposed method are still significant.

Keywords Local gravity field modelling - Quasi-geoid . GNSS-levelling data - Cauchy boundary value problem . Generalized least-squares collocation · Corrector surface

\section{Introduction}

Local (quasi-) geoids serve as reference surface for the national height system and facilitate the application of Global Navigation Satellite Systems (GNSS) for heighting. They are computed by or under contract of national authorities for a target area, which is mostly identical with the whole country. Depending on the required accuracy and spatial resolution, terrestrial and/or airborne gravity surveys are conducted to acquire the necessary gravity data over the target area. The access to gravity data of comparable quality and density outside the target area is not always guaranteed, because these data are either classified or not available for other reasons. Lack of data of comparable density and quality outside the target area reduces the accuracy of the (quasi)-geoid. Significant errors are not confined to the border of the target area, but propagate throughout the whole target area. Commonly adopted approaches to reduce these errors comprise 
the use of an existing global or continental gravity model (e.g. EGM2008, see Pavlis et al. 2008, or EGG97, see Denker and Torge 1998) or the prediction of gravity data. A further error reduction is possible by a modification of the Stokes integral kernel, which is motivated by the early works of Molodensky et al. (1962), Wong and Gore (1969) and Meissl (1971).

GNSS measurements at points with known orthometric heights or normal heights (shortly, levelling-based heights) located inside the target area, however, may be a good alternative or may offer an additional opportunity to reduce these errors provided that enough points with a good spatial coverage of the target area are available, and the accuracy is at least comparable to the accuracy of the gravimetric (quasi-) geoid. Differences between ellipsoidal heights and levelling-based heights are essentially equivalent to pointwise measurements of (quasi-) geoid heights. Over the years, the number of benchmarks observed with GNSS is continuously growing in many countries, though their number is much less than the number of available gravity anomalies. At the same time, a 1- to 2-cm precision for ellipsoidal heights is feasible provided that the occupation time is long enough and the data processing has been done carefully. This is better than the quality of the best gravimetric (quasi-) geoids. Moreover, systematic errors in levelling networks can be better controlled by GNSS, which reduces long-wavelength errors in levelling-based heights. Therefore, GNSS measurements at benchmarks of good quality and spatial coverage represent a valuable data set to reduce the errors in gravimetric (quasi-) geoids, which are caused by the lack of high-quality, dense gravity data outside the target area. In the following we will assume that such a data set is available.

When following a boundary-value approach, the determination of a local (quasi-) geoid from a combination of gravity anomalies and (quasi-) geoid heights requires a different methodology than the computation of a (quasi-) geoid from gravity anomalies alone. Prutkin and Klees (2008) have developed such a methodology. It is seen as an alternative to the commonly applied corrector-surface approach (e.g. Denker and Torge 1998; Featherstone 2000; Fotopoulos 2005; van Loon 2008) and least-squares collocation (Meissl 1976; Tscherning 1978; Moritz 1989). The main limitation of the approach of Prutkin and Klees (2008) is, however, that it does not permit to properly take random data noise into account. In fact, it is a deterministic approach. Noise is addressed indirectly by the choice of a single parameter, which determines the smoothness of the combined (quasi-) geoid model. Prutkin and Klees (2008) propose to choose this parameter equal to the average standard deviation of an observed difference between geometric and gravimetric (quasi-) geoid height. This is a serious limitation as standard deviations may vary significantly from point to point. Moreover, it is known that there are strong noise correlations among gravimetric (quasi-) geoid heights and to a lesser extent also among ellipsoidal heights and among levelling-based heights. There are also cross-correlations between gravimetric (quasi-) geoid heights and levellingbased heights, for the latter are being used when computing a gravimetric (quasi-) geoid. An example of noise correlations is shown in Fig. 2. It displays the noise covariance matrices of ellipsoidal heights, normal heights and gravimetric quasi-geoid heights at a set of benchmarks in Switzerland (cf. Sect. 3 for more details about this data set).

Here, we propose an extension of the methodology of Prutkin and Klees (2008) to noisy data with spatially varying noise variances and/or noise correlations. We study the effect of correlated noise on the combined (quasi-) geoid and compare the results with the method of Prutkin and Klees (2008), various corrector surfaces and least-squares collocation.

The paper is organized as follows: in Sect. 2, we extend the methodology of Prutkin and Klees (2008) to noisy data with full noise covariance information. Moreover, we present an alternative interpretation of the methodology of Prutkin and Klees (2008), which emphasizes the complementarity of GNSS-levelling data to gravity anomaly data if gravity anomalies of comparable quality and density outside the target area are not available. Here, 'suitable' refers to either quality or density or both of them. In Sect. 3, we introduce the data set, which is being used in this study. In Sect. 4, we apply the proposed methodology to real data. In particular, we show that when noise correlations are neglected errors up to $10 \%$ of the differences between geometric and gravimetric quasi-geoid heights can be introduced. In Sect. 5, we compare the combined (quasi-) geoid solution obtained with the methodology of Sect. 2 with various corrector surfaces. The latter are estimated from the data by least-squares. We demonstrate that there are significant errors in the corrector-surface solutions, which may take up values of several centimetres for the Swiss data set. In Sect. 6, we extend the comparison to the method of least-squares collocation with trend parameters and data noise. The trend parameters and the parameters of the signal covariance function of the differences between geometric and gravimetric (quasi-) geoid heights are estimated from the data using an iterative approach and non-linear least-squares techniques. We show that the least-squares collocation solution is closer to the solution obtained with the proposed methodology than most of the corrector-surface solutions. We close the paper with a discussion (Sect. 7) and some conclusions (Sect. 8).

\section{Methodology}

Suppose a quasi-geoid has been computed for the target area using all available gravity data. The corresponding disturbing potential is $T_{\text {grav }}$. Suppose ellipsoidal heights $h$ have been observed with GNSS at a set of benchmarks with given 
normal heights $H$ and have been converted into disturbing potential values (function $f$ ) using the normal gravity at the corresponding telluroid points. We want to use this data set to obtain a new quasi-geoid solution. The corresponding disturbing potential is $T$. Then,

$T=T_{\text {grav }}+u$,

where $u$ is the solution to the problem

$\Delta u=0$ outside $S$

$-\left.\frac{\partial u}{\partial r}\right|_{S}-\left.\frac{2}{r} u\right|_{S}=0$

$\left.u\right|_{S}=q$

Here, $S$ is the telluroid, and

$q:=f-T_{\text {grav }} \mid s$.

Note that problem (2) provides only an approximation to $u$ up to the linearization error and the error of the spherical approximation (e.g. Heck 1997). The former is negligible, because $u$ is a residual quantity. The latter can be reducted whenever necessary by applying ellipsoidal corrections.

The quantity $\frac{q}{\gamma}$, where $\gamma$ is the normal gravity at the telluroid, is the difference between the geometric height anomaly

$\zeta_{\text {geom }}:=h-H$,

and the gravimetric height anomaly

$\zeta_{\text {grav }}:=\frac{\left.T_{\text {grav }}\right|_{S}}{\gamma}$.

Unfortunately, no algorithm to compute a solution of problem (2) is known yet, because $S$ is an open surface. However, Prutkin and Klees (2008) found a numerical solution to the problem

$\Delta u=0$ outside $S$

$-\left.\frac{\partial u}{\partial r}\right|_{S}=0$

$\left.u\right|_{S}=q$

The problems (2) and (6) use different differential operators in the first boundary condition: problem (2) uses the gravity anomaly operator $-\frac{\partial}{\partial r}-\frac{2}{r}$, while problem (6) uses the gravity disturbance operator $-\frac{\partial}{\partial r}$.

The question whether problem (6) may replace problem (2) depends on the magnitude of $\left.u\right|_{S}$. As long as

$|u|_{S} \mid<\frac{r}{2} \sigma_{\Delta g}$,

where $\sigma_{\Delta g}$ is the standard deviation of the terrestrial gravity anomalies, it is safe to replace problem (2) by problem (6). To obtain an idea about the magnitude of $\left.u\right|_{S}$, we remember that $\left.u\right|_{S}$ scales with the difference between geometric and gravimetric quasi-geoid heights, and therefore is a small quantity, usually of the order of a few $\mathrm{m}^{2} / \mathrm{s}^{2}$. The accuracy of currently available surface gravity anomalies is not better than, say, $0.5 \mathrm{mGal}$; for many countries it is worse. Therefore, as long as $u$ is smaller than, say, $15 \mathrm{~m}^{2} / \mathrm{s}^{2}$, the quasi-geoid error introduced when replacing problem (2) by problem (6) is below the data noise level. Hence, as long as $u<15 \mathrm{~m}^{2} / \mathrm{s}^{2}$, problem (6) provides a good approximation to problem (2).

Prutkin and Klees (2008) suggest to represent $u$ as the mean of two harmonic functions, $u_{\text {int }}$ and $u_{\text {ext }}$, which form a Kelvin transform pair (Kellogg 1929) with respect to a sphere $S_{R}$ of radius $R$ that best fits the target area (i.e. a locally best fitting sphere). That is, if $u_{\mathrm{ext}}$ is harmonic in the domain $D_{\text {ext }} \subset \mathbb{R}^{3}$, where $D_{\text {ext }}$ is the domain outside a sphere of radius $R-d$ (the choice of $d$ will be discussed later), then the function

$u_{\text {int }}(y)=\left(\frac{R}{|y|}\right) u_{\mathrm{ext}}(x), \quad x=\frac{R^{2}}{|y|^{2}} y$,

is harmonic in the domain $D_{\text {int }}$. $D_{\text {int }}$ is obtained from $D_{\text {ext }}$ by an inversion in the sphere $S_{R}=\{x:|x|=R\}$, i.e. by the mapping $x \rightarrow y=\frac{R^{2}}{|x|^{2}} x$. If $d / R$ is small, any point on the sphere $S_{R-d}$ (which is the boundary of the domain of harmonicity of $u_{\mathrm{ext}}$ ) is mapped onto a point with distance $\left.R\left(1+d / R+O(d / R)^{2}\right)\right) \approx R+d$ from the origin, i.e. onto a point close to the surface of the sphere with radius $R+d$. Hence, if $d / R$ is small, we can consider the boundary of the domain $D_{\text {int }}$ as the surface of a sphere with radius $R+d$ without introducing significant errors in the solution. In reality, the telluroid surface $S$ differs from the surface of a locally best-fitting sphere $S_{R}$ by less than a few kilometres. If this difference is denoted $h$, the error in the quasi-geoid height we introduce when taking $u$ as the mean of the Kelvin transform pair $u_{\text {ext }}$ and $u_{\text {int }}$ is $\frac{h}{R \gamma} u$. When we set $h=5 \mathrm{~km}$, $R=6371 \mathrm{~km}$ and $u=1 \mathrm{~m}^{2} / \mathrm{s}^{2}$, the error is $<0.1 \mathrm{~mm}$.

The functions $u_{\text {ext }}$ and $u_{\text {int }}$ can be written in terms of their trace $\chi$ on the boundary of the domain of harmonicity $S_{R-d}$ and $S_{R+d}$, respectively, using Poisson's integral (e.g. Kellogg 1929) as

$u_{\mathrm{ext}}(x)=\mathscr{K}_{R-d}(\chi)(x), \quad|x|>R-h$,

$u_{\text {int }}(x)=\mathscr{K}_{R+d}(\chi)(x), \quad|x|<R+h$.

The integral operator $\mathscr{K}_{r}$ is defined as

$\mathscr{K}_{r}(\chi)(x):=\iint_{y \in S_{r}} \chi(y) K_{r}(x, y) \mathrm{d} S_{r}(y)$,

where $K_{r}(x, y)$ is the Poisson kernel for a sphere of radius $r$ :

$K_{r}(x, y)=\frac{|x|^{2}-r^{2}}{4 \pi r} \frac{1}{|x-y|^{3}}, \quad|y|=r$.

Hence, the solution to problem (6) in the vicinity of the telluroid $S$ can formally be written as

$u(x)=\frac{1}{2}\left(\mathscr{K}_{R-d}(\chi)(x)+\mathscr{K}_{R+d}(\chi)(x)\right)$. 
To obtain a numerical solution of $u$ in a 3D neighborhood of the telluroid $S$ from given values $\left.u\right|_{S}$, we need to discretize the integral (11). We select nodes $\left\{y_{i} \in S: i=1, \ldots, I\right\}$ and associated weights $w_{i}$ and approximate the integral operator (11) by

$\mathscr{K}_{r}(\chi)(x) \approx \hat{\mathscr{K}}_{r}(\chi)(x)=\sum_{i=1}^{I} \chi\left(r \hat{y}_{i}\right) K_{r}\left(x, r \hat{y}_{i}\right) r^{2} w_{i}$,

where $\left\{\hat{y}_{i}: i=1, \ldots, I\right\}$ are the nodes on the unit sphere, i.e. $\hat{y}_{i}=\frac{y_{i}}{\left|y_{i}\right|}$. The simplest choice is an equiangular grid of nodes with weights

$w_{i}=\Delta^{2} \sin \vartheta_{i}$

where $\Delta$ is the grid spacing in $\mathrm{rad}$, and $\left\{\vartheta_{i}: i=1, \ldots, I\right\}$ are the co-latitudes of the grid points. Correspondingly, we define

$\hat{u}(x):=\frac{1}{2}\left(\hat{\mathscr{K}}_{R-d}(\chi)(x)+\hat{\mathscr{K}}_{R+d}(\chi)(x)\right)$.

When we insert Eq. (14) into Eq. (16), we obtain

$$
\begin{aligned}
\hat{u}(x)= & \sum_{i=1}^{I} \chi_{i}\left[(R-d)^{2} K_{R-d}\left(x,(R-d) \hat{y}_{i}\right)\right. \\
& \left.+(R+d)^{2} K_{R+d}\left(x,(R+d) \hat{y}_{i}\right)\right] \frac{w_{i}}{2},
\end{aligned}
$$

where we have used $\chi\left((R-d) \hat{y}_{i}\right)=\chi\left((R+d) \hat{y}_{i}\right)=: \chi_{i}$. $u(x)$ is not known at all point $x \in S$, but only at the $J$ GNSSlevelling points $\left\{x_{j} \in S: j=1, \ldots, J\right\}: u_{\mid S}\left(x_{j}\right)=q\left(x_{j}\right)$ (for simplicity reasons we use the same notation for points on the Earth's surface and on the telluroid). Hence, the fully discretized observation equations are up to the discretization error

$$
\begin{aligned}
q\left(x_{j}\right)= & \sum_{i=1}^{I} \chi_{i}\left[K_{R-d}\left(x_{j},(R-d) \hat{y}_{i}\right)(R-d)^{2}\right. \\
& \left.+K_{R+d}\left(x_{j},(R+d) \hat{y}_{i}\right)(R+d)^{2}\right] \frac{w_{i}}{2} .
\end{aligned}
$$

Equation (18) is a system of $J$ linear equations with $I$ unknowns. We define vectors $\mathbf{q}=\left(q\left(x_{j}\right)\right)$ and $\chi=\left(\chi_{i}\right)$ and a matrix

$$
\begin{aligned}
\mathbf{K}= & \left(K_{j, i}\right)=\left(\left[(R-d)^{2} K_{R-d}\left(x_{j},(R-d) \hat{y}_{i}\right)\right.\right. \\
& \left.\left.+(R+d)^{2} K_{R+d}\left(x_{j},(R+d) \hat{y}_{i}\right)\right] \frac{w_{i}}{2}\right),
\end{aligned}
$$

and write Eq. (18) in matrix-vector notation as

$\mathbf{q}=\mathbf{K} \chi$.

In the presence of noise, we write the last equation as a Gauss-Markov model

$E\{\mathbf{q}\}=\mathbf{K} \chi, \quad D\{\mathbf{q}\}=\mathbf{C}_{n}$, where $E\{\cdot\}$ and $D\{\cdot\}$ are the expectation and dispersion operator, respectively. The noise covariance matrix $\mathbf{C}_{n}$ is the sum of the noise covariance matrices of the gravimetric quasigeoid heights $\zeta_{\text {grav }}$, the ellipsoidal heights $h$ and the normal heights $H$. Adding a random vector of errors $\varepsilon=\left(\varepsilon_{j}\right)$, we obtain

$\mathbf{q}+\boldsymbol{\varepsilon}=\mathbf{K} \chi, \quad E\{\boldsymbol{\varepsilon}\}=\mathbf{0}, \quad D\{\boldsymbol{\varepsilon}\}=D\{\mathbf{q}\}=\mathbf{C}_{n}$.

Assuming $\operatorname{rank} \mathbf{K}=J$ and $\mathbf{C}_{n}$ positive definite, the leastsquares solution to Eq. (22) is

$\hat{\chi}=\left(\mathbf{K}^{T} \mathbf{C}_{n}^{-1} \mathbf{K}\right)^{-1} \mathbf{K}^{T} \mathbf{C}_{n}^{-1} \mathbf{q}$.

To control the amplification of data noise, some regularization may be needed though the discretization already acts as a smoother. Then, Eq. (23) is replaced by the regularized least-squares solution

$\hat{\chi}_{\alpha}=\left(\mathbf{K}^{T} \mathbf{C}_{n}^{-1} \mathbf{K}+\alpha \mathbf{R}\right)^{-1} \mathbf{K}^{T} \mathbf{C}_{n}^{-1} \mathbf{q}$,

where $\mathbf{R}$ is the regularization matrix and $\alpha$ is the regularization parameter. Once the solution $\hat{\chi}_{\alpha}$ has been computed, the regularized least-squares solution $\hat{u}_{\alpha}$ to problem (6) on $S$ and within a small neighbourhood of $S$ is

$\hat{u}_{\alpha}(x)=\hat{\chi}_{\alpha}^{T} \mathbf{k}(x)$,

where $\mathbf{k}(x)$ is the $I \times 1$ vector with elements

$$
\begin{aligned}
k\left(x, \hat{y}_{i}\right)= & \left((R-d)^{2} K_{R-d}\left(x,(R-d) \hat{y}_{i}\right)\right. \\
& \left.+(R+d)^{2} K_{R+d}\left(x,(R+d) \hat{y}_{i}\right)\right) \frac{w_{i}}{2} .
\end{aligned}
$$

Once $\hat{u}_{\alpha}(x)$ has been computed at points $x \in S$, we obtain an estimate of the disturbing potential $T$, Eq. (1),

$\hat{T}_{\alpha}(x)=T_{\text {grav }}+\hat{u}_{\alpha}(x), \quad x \in S$,

and an estimate of the height anomaly,

$\hat{\zeta}_{\alpha}(x)=\zeta_{\text {grav }}(x)+\frac{\hat{u}_{\alpha}(x)}{\gamma(x)}, \quad x \in S$.

The solution to problem (6) requires three choices to be made: (1) the weights and nodes of the cubature formula that are being used to discretize the model (14); (2) the depth $d$, Eqs. (9) and (10); and (3) the regularization parameter $\alpha$, Eq. (24). We choose a simple discretization scheme, which uses the nodes of an equiangular grid with grid spacing $\Delta$ and weights according to Eq. (15). To obtain an overdetermined system of linear equations, Eq. (18), the number of grid points must be smaller than the number of GNSSlevelling points. To guarantee this, we choose the grid spacing $\Delta$ as function of the mean distance between the GNSS-levelling points, $s$, according to

$\Delta<\frac{s}{R} \sqrt{\sin \vartheta_{0}}$ 
where $R$ is the radius of the locally best-fitting sphere $S_{R}$, and $\vartheta_{0}$ is the mean co-latitude of the target area. The depth $d$ and the regularization parameter $\alpha$ control the smoothness of the solution and the fit of the model to the data.

An optimal solution could be found by searching for the optimal pair $(d, \alpha)$, for instance using Generalized Cross Validation (GCV) (Craven and Wahba 1979). We use a somewhat simpler approach to find a suitable pair $(d, \alpha)$ : we solve problem (6) for a range of depths; for each depth, we determine the optimal regularization parameter using GCV. The minimum of the GCV functional is found using the algorithm of Brent (1973). The pair for which the RMS of the leastsquares residuals is close to the (average) standard deviation of the geometric quasi-geoid heights at the GNSS-levelling points is the selected one. This approach is numerically not that expensive, because the search space of candidate depths does not need to be sampled very densely as will be shown in Sect. 4. Note that there can be more than one pair that fulfils the minimum RMS criterion. Empirically, we found that if there are several pairs $(d, \alpha)$, the corresponding solutions differ by just a few millimetres, i.e. they are essentially identical in the presence of data noise.

\section{Test data set}

To demonstrate the performance of the proposed methodology, we use a data set which covers the entire Switzerland. It has already been used in Fotopoulos (2005) and van Loon (2008) to investigate the use of Variance Component Estimation (VCE) techniques to estimate variance factors for the noise covariance matrices of geoid heights, ellipsoidal heights and orthometric heights assuming that the systematic differences between geometric and gravimetric geoid heights can be modelled using low-degree algebraic or trigonometric polynomials. Instead of geoid heights and orthometric heights as being used in Fotopoulos (2005) and van Loon (2008), we use quasi-geoid heights and normal heights, which are also available at the benchmarks observed by GNSS.

The original data set comprises ellipsoidal heights, normal heights and quasi-geoid heights at 111 levelling benchmarks covering the entire country (cf. Fig. 1). We do not use the data of the stations Zimmerwald and GR3 318. Station Zimmerwald was held fixed in the adjustment of the levelling network and the geoid computation. Station GR3 318 has been identified as an outlier (van Loon 2008). Hence, all computations were made using 109 data points. The area of Switzerland is about $42,000 \mathrm{~km}^{2}$, which corresponds to an average distance between the GNSS-levelling points of about $20 \mathrm{~km}$. The differences between geometric quasigeoid heights (ellipsoidal heights minus normal heights) and gravimetric quasi-geoid heights (computed from gravity

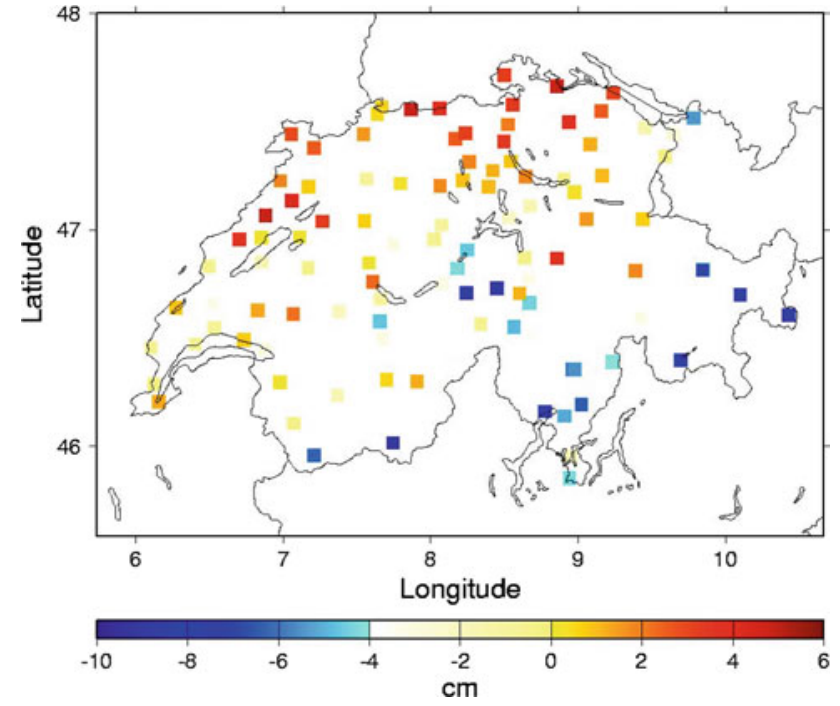

Fig. 1 Spatial distribution of the GNSS-levelling points and differences between geometric quasi-geoid heights (from GNSS at benchmarks) and gravimetric quasi-geoid heights (from gravity anomalies)

anomalies using least-squares collocation) vary between $-10 \mathrm{~cm}$ and $+6 \mathrm{~cm}$, and the RMS difference is $4 \mathrm{~cm}$. The differences are very small compared with the situation in many other countries. We explain this partially by the fact that high-quality gravity data outside Switzerland have been used in the computation of the quasi-geoid. In Sects. 4, 5 and 6 , different solutions will be compared with each other. The differences between these solutions scale proportional to the differences between geometric and gravimetric quasigeoid heights. One needs to keep this in mind when analysing the magnitude of differences between various solutions and judging whether the differences are significant or not.

Fully populated noise covariance matrices are available for ellipsoidal heights, normal heights and gravimetric quasigeoid heights (cf. Fig. 2). The noise covariance matrix of normal heights comes directly from the national adjustment of all first-order and second-order levelling measurements, which was done in geopotential numbers. The fully populated noise covariance matrix of the gravimetric quasi-geoid heights at the GNSS-levelling benchmarks was obtained by a straightforward application of error propagation to the leastsquares collocation equations. Unfortunately, omission and commission errors of the global reference gravity model have not been incorporated in the noise covariance matrix of gravimetric quasi-geoid heights.

The noise covariance matrix of the differences between geometric and gravimetric quasi-geoid heights, $\tilde{\mathbf{C}}_{n}$, can be written as

$\tilde{\mathbf{C}}_{n}=\sigma_{h}^{2} \mathbf{Q}_{h}+\sigma_{\zeta}^{2} \mathbf{Q}_{\zeta}+\sigma_{H}^{2} \mathbf{Q}_{H}$,

where $\mathbf{Q}_{h}, \mathbf{Q}_{\zeta}$ and $\mathbf{Q}_{H}$ are the cofactor matrices of, respectively, the ellipsoidal heights, the gravimetric quasi-geoid 
Fig. 2 Magnitude of elements (units of $\mathrm{cm}^{2}, \log 10$ scale from 0.01 to $25 \mathrm{~cm}^{2}$ ) of various noise covariance matrices (from left to right, top to bottom): noise covariance matrix for ellipsoidal heights, $\mathbf{C}_{h}$, normal heights, $\mathbf{C}_{H}$, gravimetric quasi-geoid heights, $\mathbf{C}_{\zeta}$, geometric quasi-geoid heights, $\mathbf{C}_{h}+\mathbf{C}_{H}$ and for the differences between geometric and gravimetric quasi-geoid heights, $\tilde{\mathbf{C}}_{n}=\mathbf{C}_{h}+\mathbf{C}_{H}+\mathbf{C}_{\zeta}$
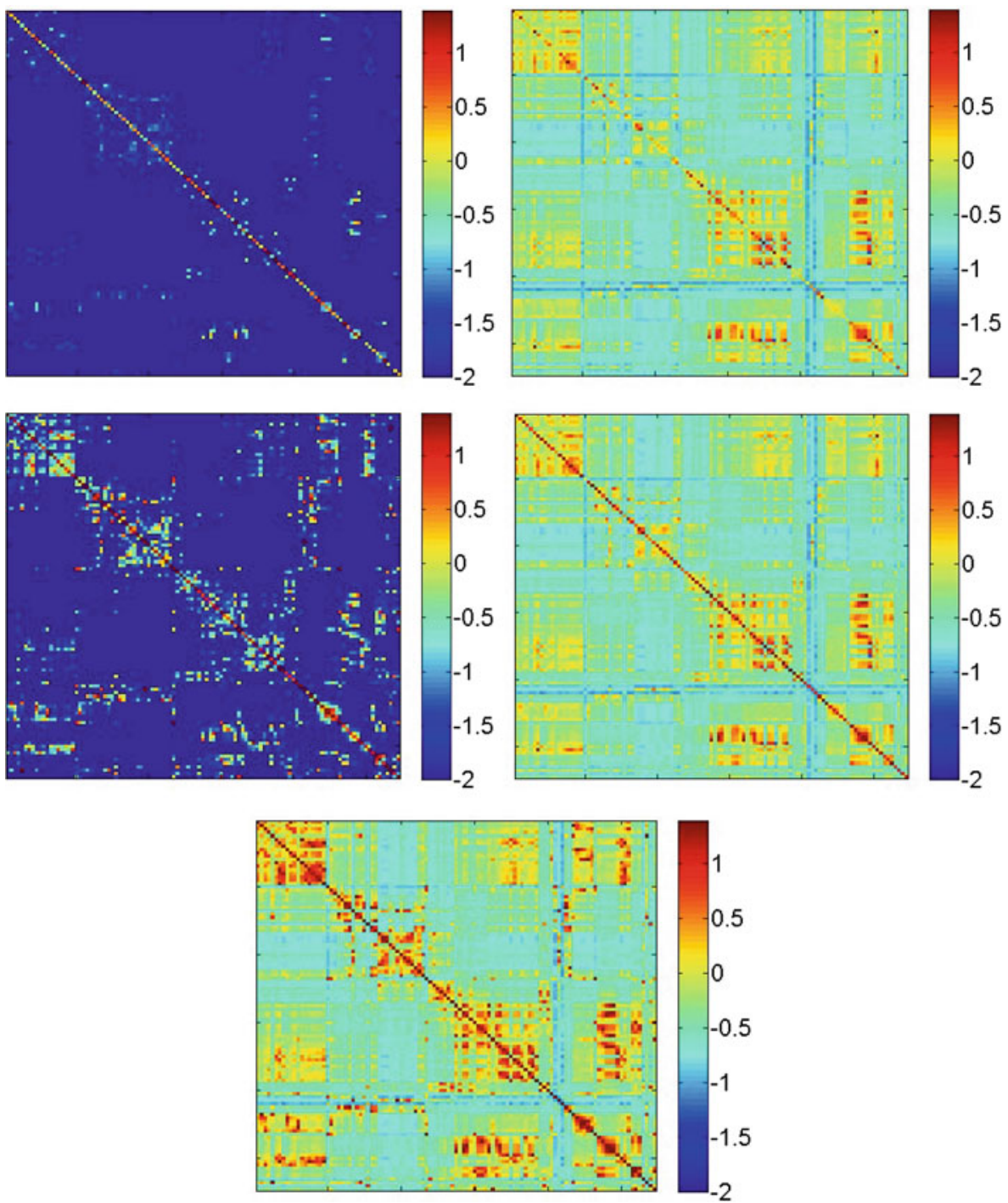

The noise covariance matrices $\tilde{\mathbf{C}}_{n}$ and $\mathbf{C}_{n}$ reveal the presence of strong correlations, in particular between nearby stations. This is mainly due to the presence of strongly correlated noise in normal heights and, though to a lesser extent, in gravimetric quasi-geoid heights. Compared with them, noise correlations between ellipsoidal heights are negligible. Table 1 provides some statistics of the main diagonal elements of the various noise covariance matrices. Note that gravimetric quasi-geoid heights and geometric quasi-geoid heights are of comparable quality; the standard deviations of the geometric quasi-geoid heights vary between $\pm 1.0 \mathrm{~cm}$ and $\pm 3.4 \mathrm{~cm}$ (average $\pm 2.0 \mathrm{~cm}$ ), and the standard deviations of the gravimetric quasi-geoid heights vary between $\pm 1.2 \mathrm{~cm}$ and $\pm 4.4 \mathrm{~cm}$ (average $\pm 2.0 \mathrm{~cm}$ ). The standard deviations of the differences between geometric quasi-geoid heights and gravimetric quasi-geoid heights vary between $\pm 1.9 \mathrm{~cm}$ and $\pm 5.4 \mathrm{~cm}$. As expected, the gravimetric quasi-geoid heights 
Table 1 Statistics of the main diagonal elements of the noise covariance matrices of GNSS ellipsoidal heights $\mathbf{C}_{e}$, normal heights $\mathbf{C}_{H}$, gravimetric quasi-geoid heights $\mathbf{C}_{\zeta}$, geometric quasi-geoid heights $\mathbf{C}_{e}+\mathbf{C}_{H}$ and the sum of the three noise covariance matrices $\mathbf{C}_{n}$

\begin{tabular}{llcccr}
\hline & $\begin{array}{l}\text { Ellipsoidal } \\
\text { heights }\end{array}$ & $\begin{array}{l}\text { Quasi-geoid } \\
\text { heights }\end{array}$ & $\begin{array}{l}\text { Normal } \\
\text { heights }\end{array}$ & $\begin{array}{l}\text { Geometric } \\
\text { quasi-geoid } \\
\text { heights }\end{array}$ & $\mathbf{C}_{n}$ \\
\hline Min & 0.08 & 1.40 & 0.65 & 1.08 & 3.89 \\
Max & 7.40 & 19.09 & 6.51 & 11.65 & 28.75 \\
Mean & 1.85 & 4.18 & 2.00 & 3.85 & 8.03 \\
\hline
\end{tabular}

Units in $\mathrm{cm}^{2}$

contribute the most to the overall standard deviation with an average contribution of about $\pm 2 \mathrm{~cm}$.

For the Swiss data set, the average distance between the GNSS-levelling points is $s=20 \mathrm{~km}$ and the radius of the locally best-fitting sphere is $R=6365 \mathrm{~km}$. With $\vartheta_{0}=43^{\circ}$, Eq. (29) provides an upper limit to the grid size $\Delta: \Delta<16^{\prime}$. We use $\Delta=15^{\prime}$ and obtain 83 nodes located inside the target area; hence the redundancy is $109-83=26$.

\section{Results}

\subsection{The optimal solution to problem (6)}

The first experiment is conducted in order to find the optimal pair $(d, \alpha)$ and to investigate the effect of different choices of $(d, \alpha)$ on the smoothness of the innovation function $\hat{u}_{\alpha}$, Eq. (25). The full noise covariance matrix $\mathbf{C}_{n}$ has been used in these computations. We computed a series of innovation functions for various choices of the depth $d$. Three of them are shown in Fig. 3: $d=50 \mathrm{~km}, d=100 \mathrm{~km}$ and $d=200 \mathrm{~km}$. The statistics of the residuals of the least-squares adjustment and the corresponding regularization parameters are given in Table 2 together with the corresponding information for some other depths.

The range of the residuals of the least-squares adjustment and the RMS of the residuals is essentially the same for the three depths. However, a visual inspection of the solutions reveals some significant differences in the spatial pattern of the innovation function $\hat{u}_{\alpha}$. Solution $d=50 \mathrm{~km}$ is characterized by larger gradients in the neighbourhood of the GNSS-levelling points. These local features are likely caused by the influence of data noise, which is amplified when the data are downward continued. This is not corrected for by the regularization term, though the $d=50 \mathrm{~km}$ solution has the largest regularization parameter among all solutions. The solution $d=200 \mathrm{~km}$ is obviously too smooth. The a-posteriori RMS fit to the data is $\pm 2.2 \mathrm{~cm}$, which is above the (average) standard deviation of the geometric quasi-geoid heights $( \pm 2.0 \mathrm{~cm})$. We prefer solutions which have a RMS fit to the data not exceeding and being close to the (averaged) standard deviation of the geometric quasi-geoid heights. When taking this as a criterion for optimality, the optimal solution is found for a depth between $d=100 \mathrm{~km}$ and $d=130 \mathrm{~km}$. The innovation functions for this range of depths are very much alike with maximum differences below $0.5 \mathrm{~cm}$. Hence, the solution is not that sensitive to the choice of the depth over this range of depths. For the rest of the study, we choose the solution $d=120 \mathrm{~km}$ with full noise covariance matrix as the reference solution. It is as smooth as the other solutions over this range of depths, but has the smallest effective regularization parameter. The effective regularization parameter is defined as the ratio of the regularization parameter and the mean amplitude of the main diagonal elements of the normal equation matrix. The latter strongly depends on the choice of the depth. The reference solution is not shown in Fig. 3, because, visually, there is no difference with respect to the $d=100 \mathrm{~km}$ solution.

\subsection{Comparison with Prutkin and Klees (2008)}

The second experiment has been designed to quantify the differences between the approach by Prutkin and Klees (2008) and the methodology presented in Sect. 2. The approach by Prutkin and Klees (2008) assumes a square system matrix K and solves in fact Eq. (20), or its regularized version, $\mathbf{q}=$ $(\mathbf{K}+\alpha \mathbf{I}) \chi$. The advantage of a square system matrix is its better condition number compared with the condition number of the normal matrix. Remember that the system matrix represents a discretized version of the first-kind Poisson integral equation, i.e. it is ill-conditioned, and its condition number increases with increasing number of nodes. A square system matrix implies that the Poisson integral equation on the surface of a sphere with radius $R-d$ needs to be discretized at a number of nodes, which is equal to the number of data points. Therefore, it is not possible to use an equiangular grid as proposed in Sect. 2, because then the number of nodes will not be the same as the number of data points. There are two possibilities to realize a square system matrix: (1) the Poisson integral equation is discretized at the data points; or (2) the data are interpolated at the nodes of an equiangular grid, and the interpolated data are used as 'observations'; in order to avoid an artificial increase of the number of observations, the size of the grid is chosen such that the number of nodes is as close as possible to the number of data points.

We compute two solutions according to the approach by Prutkin and Klees (2008). Solution 1 uses the data points as nodes to discretize the Poisson integral equation, and solution 2 uses a 15' equiangular grid as nodes. The latter comprises 83 nodes inside the target area. This grid is the same grid as is being used when computing the solution with the proposed methodology of Sect. 2. To obtain a square system matrix $\mathbf{K}$, the original data given at the GNSS-levelling points are 
Fig. 3 Various solutions to problem (6) with full noise covariance matrix: from left to right and top to bottom: depth $d=50 \mathrm{~km}, d=100 \mathrm{~km}$, $d=200 \mathrm{~km}$, difference between $d=100 \mathrm{~km}$ and $d=50 \mathrm{~km}$ and differences between

$d=100 \mathrm{~km}$ and $d=200 \mathrm{~km}$. For every depth $d$, the optimal regularization parameter has been determined with GCV
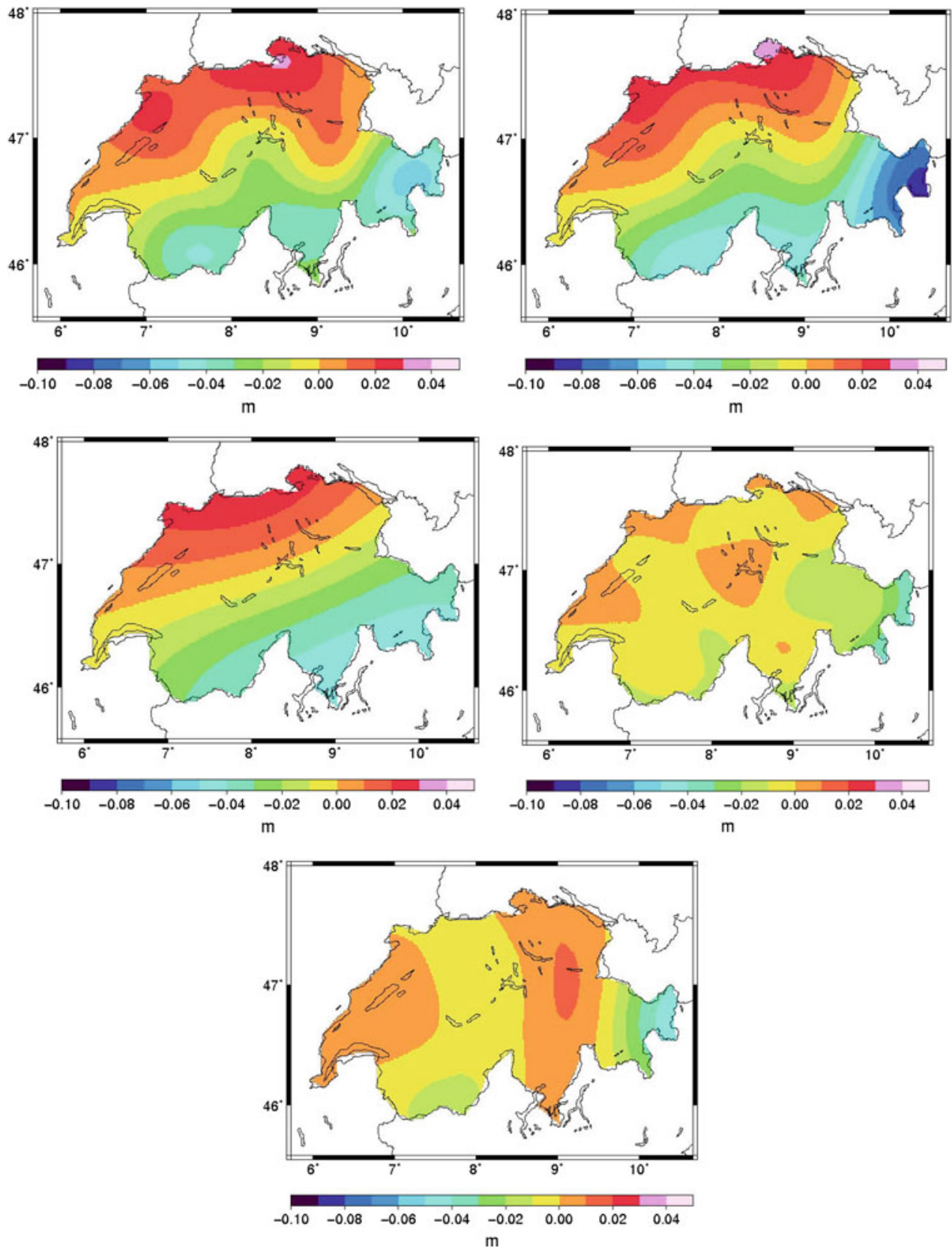

interpolated at the nodes of the $15^{\prime}$ equiangular grid using the GMT-routine 'surface' with tension factor 1 (Smith and Wessel 1990; Wessel and Smith 1991). The choice of a tension factor 1 is justified in Sect. 5. The trade-off between the fit to the data and the smoothness of the solution depends on the depth $d$ and the regularization parameter $\alpha$. We found that different pairs $(d, \alpha)$ provide solutions which differ by less than a few millimetres. After several experiments, we select a depth of $d=100 \mathrm{~km}$ for both solutions. The regularization parameter for solution 1 is $\alpha=0.005$; the effective regularization parameter is 0.6101 , and the RMS fit to the data is $1.8 \mathrm{~cm}$. The regularization parameter for solution 2 is $\alpha=0.001$; the effective regularization parameter is 0.1219 , and the RMS fit to the data is $1.9 \mathrm{~cm}$.

Figure 4 shows the two solutions and the differences with respect to the reference solution (i.e. the solution, which is obtained with the proposed methodology and the full noise covariance matrix). Solution 2 is closer to the reference solution than solution 1, which is due to a better fit in the eastern part of Switzerland. The differences with respect to the reference solution vary between -1.2 and $1.2 \mathrm{~cm}$; the RMS difference is $0.5 \mathrm{~cm}$. We explain the larger differences between the reference solution and solution 1 in the eastern part of Switzerland with the effect of the interpolation of the data at 
Table 2 Statistics of the least-squares residuals of the solution to problem (6) for various choices of the depth $d$

\begin{tabular}{llllll}
\hline$d(\mathrm{~km})$ & Min & Max & Mean & RMS & $\begin{array}{l}\text { Effective } \\
\text { regularization } \\
\text { parameter }\end{array}$ \\
\hline 50 & -5.4 & 4.4 & -0.5 & 2.0 & 0.966 \\
75 & -4.8 & 4.9 & -0.3 & 1.9 & 0.315 \\
100 & -4.7 & 5.1 & -0.2 & 1.9 & 0.111 \\
120 & -4.8 & 5.3 & -0.2 & 2.0 & 0.062 \\
150 & -5.4 & 5.7 & -0.3 & 2.1 & 0.250 \\
200 & -5.7 & 6.0 & -0.2 & 2.2 & 0.359 \\
\hline
\end{tabular}

Units in $\mathrm{cm}$. The last column shows the effective regularization parameter $\alpha_{\mathrm{gcv}, \text { eff }}=\frac{\alpha_{\mathrm{gcv}}}{D}$, where $D$ is the average diagonal element of the matrix $\mathbf{K}^{T} \mathbf{C}_{n}^{-1} \mathbf{K}$

the nodes of the 15' equiangular grid. The 'observed' difference between geometric and gravimetric quasi-geoid heights at the two most eastern GNSS-levelling benchmarks is -7.1 and $-9.9 \mathrm{~cm}$, respectively (cf. Fig. 1); the interpolated value at the closest node of the 15' equiangular grid (distance to the data point is 11.7 and $6.3 \mathrm{~km}$, respectively) is only -5.4 and $-7.3 \mathrm{~cm}$, respectively. Hence, one must be careful when interpolated data is being used as 'observations' in the further data processing.
4.3 Effect of noise correlations and spatially varying noise variances

A third experiment has been designed to investigate the effect of noise correlations on the innovation function $\hat{u}_{\alpha}$, Eq. (25). In practice, fully populated noise covariance matrices for ellipsoidal heights, normal heights, and gravimetric quasi-geoid heights at GNSS-levelling benchmarks are rarely available. More often than not, correlations are ignored, and diagonal covariance matrices are used, which only account for spatially varying variances. Sometimes, even the latter are not available, and the noise covariance matrix $\mathbf{C}_{n}$ is assumed to be a (scaled) unit matrix. To test the effect on the innovation function of noise correlations and spatially varying noise variances in differences between geometric and gravimetric quasi-geoid heights, several computations were done with a fully populated, a diagonal and a (scaled) unit noise covariance matrix $\mathbf{C}_{n}$. All computations use the same depth of $d=120 \mathrm{~km}$. For every choice of the noise covariance matrix, the regularization parameter is determined using GCV. Figure 5 shows the differences with respect to the reference solution (solution with depth $d=120 \mathrm{~km}$ and full noise covariance matrix), and Table 3 shows the statistics of the differences.
Fig. 4 Innovation function according to Prutkin and Klees (2008) $(d=100 \mathrm{~km})($ left column) and differences with respect to the reference solution (proposed methodology with full noise covariance matrix) (right column). First row data interpolated at the nodes of a 15- min grid; second row original data used
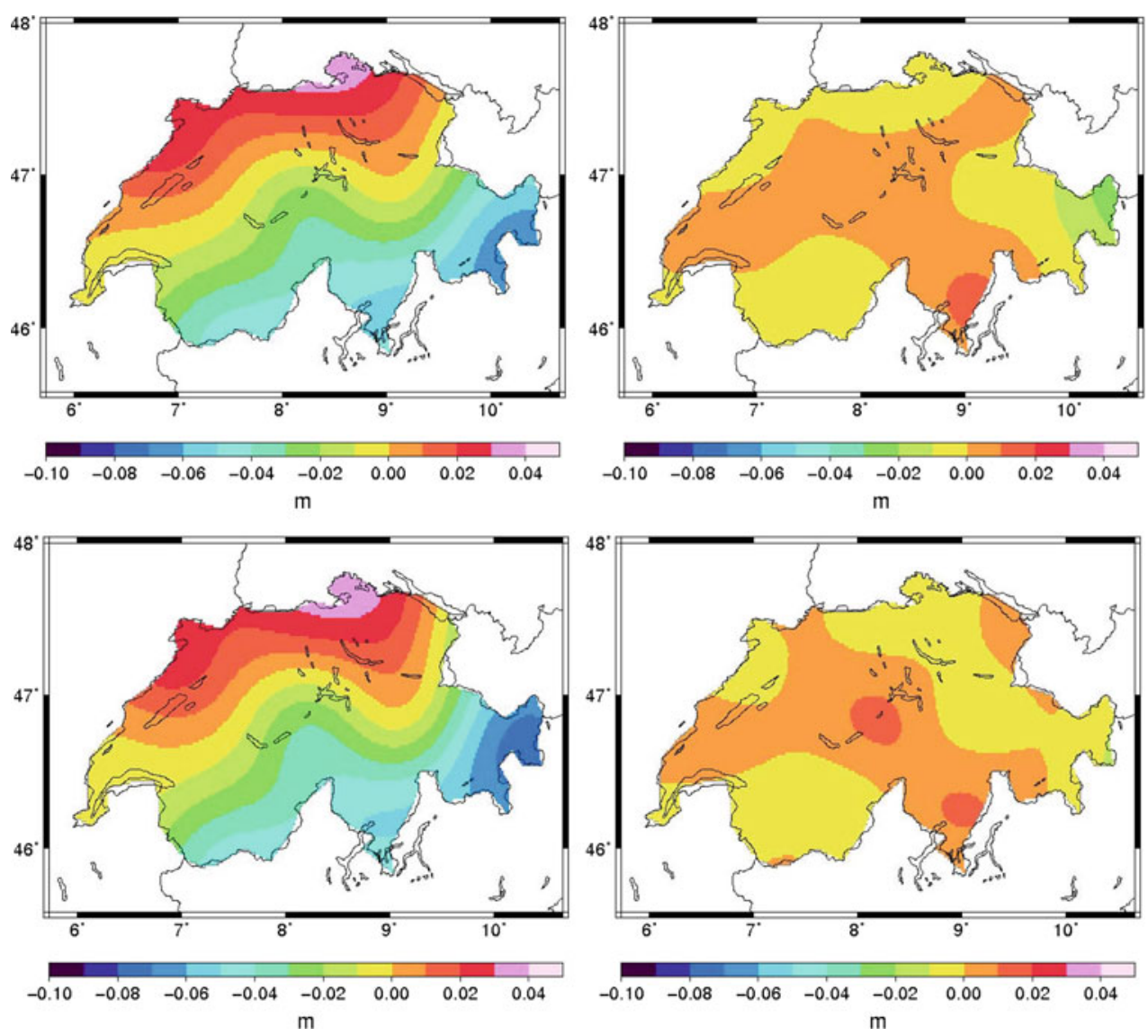
Fig. 5 Innovation functions computed with a simplified noise covariance matrix. Top left panel diagonal noise covariance matrix (i.e. noise correlations have been neglected); bottom left panel unit noise covariance matrix (i.e. noise correlations and spatial variations of noise variances have been neglected). Right column corresponding differences with respect to the reference solution (proposed methodology with a full noise covariance matrix). All solutions have been computed for a depth of $d=120 \mathrm{~km}$; the regularization parameter has been determined with GCV
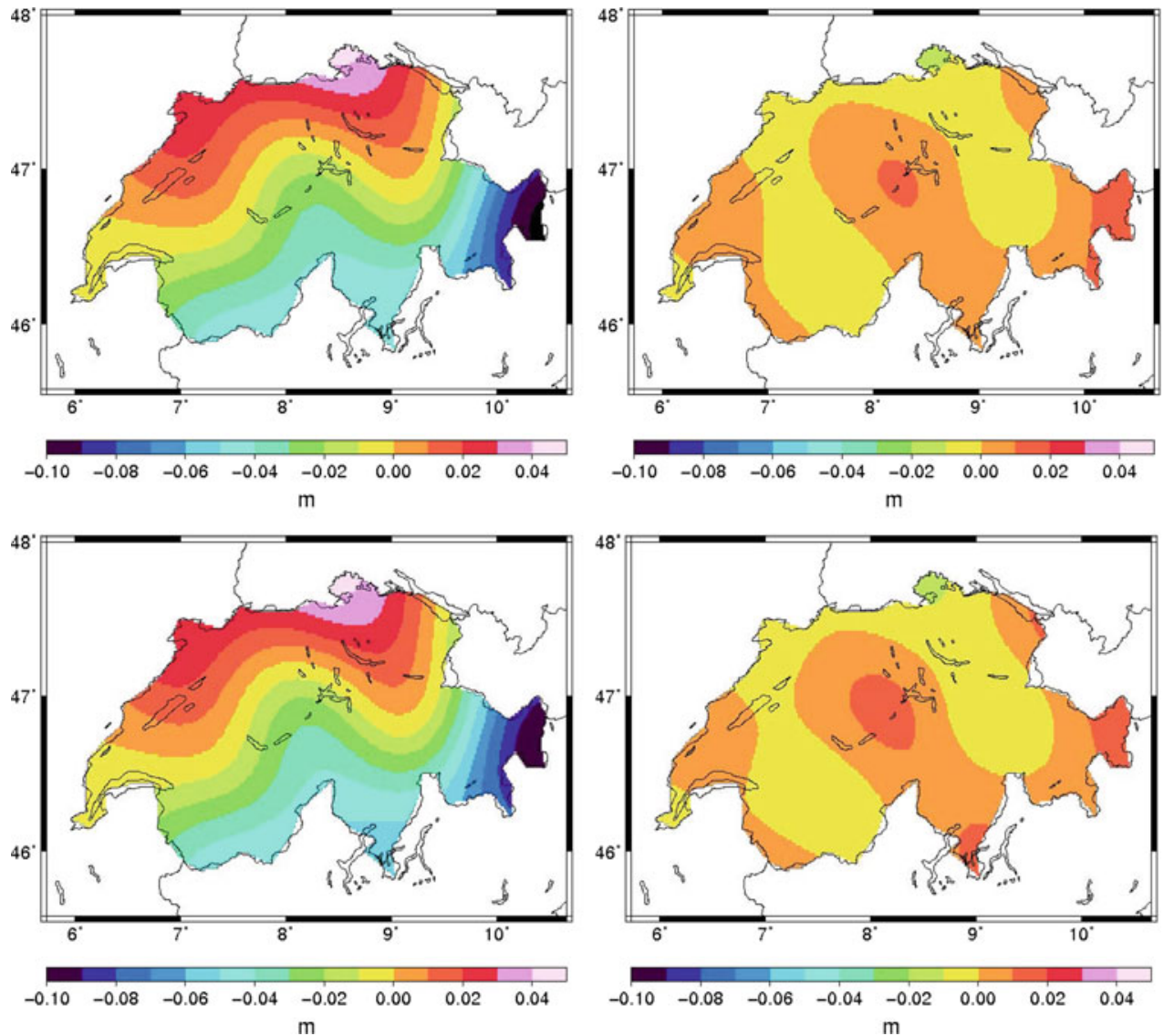

Table 3 Statistics of the differences with respect to the reference solution (proposed methodology with full noise covariance matrix) of four innovation function solutions

\begin{tabular}{lccccc}
\hline Approach & Min & Max & Mean & RMS & SD \\
\hline $\begin{array}{l}\text { Prutkin and Klees (2008); } \\
\text { gridded data }\end{array}$ & -2.5 & 1.2 & 0.0 & 0.5 & 0.5 \\
$\begin{array}{l}\text { Prutkin and Klees (2008); } \\
\quad \text { original data }\end{array}$ & -1.2 & 1.2 & 0.0 & 0.5 & 0.5 \\
$\begin{array}{l}\text { Proposed methodology; } \\
\text { diagonal noise }\end{array}$ & -0.5 & 1.1 & 0.2 & 0.4 & 0.3 \\
$\begin{array}{c}\text { covariance matrix } \\
\begin{array}{c}\text { Proposed methodology; } \\
\text { unit noise covariance } \\
\text { matrix }\end{array}\end{array}$ & -0.5 & 1.7 & 0.3 & 0.6 & 0.5 \\
\hline
\end{tabular}

The statistics are computed at a 1-min equiangular grid restricted to the target area. Units in $\mathrm{cm}$

Neglecting correlations introduces errors varying between -1.2 and $1.9 \mathrm{~cm}$. These errors are systematic and positive for most parts of the country (cf. Fig. 5). A scaled unit matrix leads to similar error amplitudes and patterns (cf. Fig. 5). The differences between the solution with a diagonal noise covariance matrix and a scaled unit matrix are below $0.4 \mathrm{~cm}$, though the noise variances vary significantly from point to point (between 3.9 and $28.8 \mathrm{~cm}^{2}$ ). This indicates that for the Swiss data set it is more critical to neglect noise correlations than to ignore spatially varying noise variances. This can be explained by the strong correlations present in the noise covariance matrix $\mathbf{C}_{n}$. Such strong noise correlations are not a special property of the Swiss data set, but typical for all noise covariance matrices of differences between geometric and gravimetric quasi-geoid heights. Remarkable is that the errors introduced in the innovation function when neglecting correlations or neglecting spatial variations of the noise variances lead to large errors in the eastern part of Switzerland. Obviously, when taking noise correlations and spatially varying noise variances into account as the reference solution does, these two data points are downweighted, i.e. they contribute less to the solution. Vice versa, if noise correlations are neglected or spatially varying noise variances are ignored, too high weights are assigned to them, which explains the large differences relative to the reference solution.

\section{Comparison with the corrector-surface approach}

The next experiment was conducted to test the performance of the corrector-surface approach, which is the most popular method to deal with differences between geometric and gravimetric quasi-geoid heights. Originally, corrector surfaces were used to facilitate the transformation of ellipsoidal 
heights into levelling-based heights (e.g. Featherstone 1998; Grebenitcharsky et al. 2005; Nahavandchi and Soltanpour 2006). However, they are also being used as a simple tool to obtain improved quasi-geoid models from a combination of gravity anomalies and GNSS-levelling data (e.g. de Min 1996; de Bruijne et al. 1997; Featherstone 2000). A practical advantage of this simple approach is that no new quasi-geoid needs to be computed if new GNSS measurements at benchmarks become available. The innovation function obtained with a full noise covariance matrix $\mathbf{C}_{n}$ and a depth of $d=$ $120 \mathrm{~km}$ serves as the reference solution.

The type of the corrector surface may be suggested by the geometry of the problem. For instance, the popular model of Heiskanen and Moritz (1967) has been developed to account for different reference ellipsoids to which GNSS ellipsoidal heights and levelling-based heights may refer. However, in most applications, there is no information available in favour of a particular type of an analytical corrector surface. Typically, low-order polynomials are preferred, because the differences between geometric and gravimetric quasi-geoid heights are usually associated with a smoothly varying function. Moreover, the mean distance between GPS-levelling points poses a lower limit to the wavelengths of the corrections that can be determined. We follow the same idea and analyse various corrector surface models within the class of models

$$
\begin{aligned}
u(\varphi, \lambda)= & \sum_{i=0}^{N} \sum_{j=0}^{N} p_{i j} \varphi^{i}(\lambda \cos \varphi)^{j}+q_{0}+q_{1} \cos \varphi \cos \lambda \\
& +q_{2} \cos \varphi \sin \lambda+q_{3} \sin \varphi+q_{4} \sin ^{2} \varphi
\end{aligned}
$$

This class of models comprises some of the most widely used corrector surface models including the model of Heiskanen and Moritz (1967). The parameters $p_{i j}$ and $q_{k}$ are estimated by least-squares following the approach of van Loon (2008): we use full noise covariance matrices for gravimetric quasi-geoid heights, GNSS ellipsoidal heights and normal heights, respectively; for every choice of the corrector surface model, optimal scaling factors are estimated for the three noise covariance matrices using VCE; the parameters of the corrector surface model are tested for significance; statistically insignificant parameters are removed from the model, and the procedure is repeated until a final set of statistically significant parameters has been found. Among the accepted models, the model with the highest level of significance has been selected. It turned out to be the model

$u(\vartheta, \lambda)=p_{01}(\lambda \cos \varphi)+p_{11}(\varphi \lambda \cos \varphi)+q_{2}(\cos \varphi \sin \lambda)$.

This model has been compared with the reference solution (cf. Fig. 6; Table 4).
The corrector surface (34) is a very smooth function, being close to a tilted plane. Therefore, it cannot model local features of differences between geometric and gravimetric quasi-geoid heights, which differ from a tilted plane. Correspondingly, the largest differences with respect to the reference solution occur in those areas where the innovation function shows larger gradients; the maximum difference is $4.3 \mathrm{~cm}$ and is located in the eastern part of Switzerland (cf. Fig. 6). The model (34) is also the only one considered in this study that has a bias $(0.6 \mathrm{~cm})$. An attempt to account for the bias by extending the model (34) fails in the sense that then only the parameter $p_{11}$ passes the test of significance.

An easy-to-get corrector surface is the minimum-curvature spline under tension (e.g. Smith and Wessel 1990) in combination with a spatial low-pass filter. The GMT software package (Wessel and Smith 1991) provides the routine 'blockmean', which does the low-pass filtering, and the routine 'surface', which computes the spline under tension. The smoothness of the spline is controlled by a tension factor, which has to be selected by the user. The default tension factor in routine 'surface' is 0.25 . We use a tension factor equal to 1 , because only then the minimum-curvature spline is a harmonic function in the $(\varphi, \lambda)$-domain. Prutkin and Klees (2008) have shown that the solution of the LaplaceBeltrami equation on the sphere (which uses extrapolated differences between geometric and gravimetric quasi-geoid heights along the border of the target area) provides a good approximation to the solution of problem (6) if the target area is not too large. Though the minimum-curvature spline with tension factor 1 does not fulfil the Laplace-Beltrami equation exactly, it is a smooth function without maxima or minima inside the target area, and hence may provide an appealing corrector surface. The solution and its difference to the reference solution are shown in Fig. 6 and Table 4. The minimumcurvature spline with tension factor 1 performs better than the model (34). The maximum differences with respect to the reference solution concentrate to an area east of $10^{\circ}$ longitude and a small area along the north-western border of Switzerland (cf. Fig. 6). For most parts of the country, the differences do not exceed $1.0 \mathrm{~cm}$.

We also tested a least-squares radial basis function approximation as corrector surface model. The parameters of a thinplate spline radial basis function model,

$$
\begin{aligned}
& q(x, \mathbf{p})=\sum_{m=1}^{M} p_{m} f\left(x, y_{m}\right), \\
& f\left(x, y_{m}\right)=\left\{\begin{array}{ll}
\left|x-y_{m}\right|^{2}\left(\ln \left(\left|x-y_{m}\right|\right)-1\right) & \text { for }\left|x-y_{m}\right|>0 \\
0 & \text { for }\left|x-y_{m}\right|=0
\end{array},\right.
\end{aligned}
$$

are computed such that $\Phi(\mathbf{p})=(\mathbf{q}-\mathbf{A} \mathbf{p})^{T} \mathbf{C}_{n}^{-1}(\mathbf{q}-\mathbf{A} \mathbf{p})+$ $\frac{1}{\alpha} \mathbf{p}^{T} \mathbf{p}$ is minimized, where $\mathbf{A}$ is the design matrix with 
Fig. 6 Various

correction-surface solutions (left column) and their difference with respect to the reference solution (proposed methodology with full noise covariance matrix) (right column). From top to bottom: corrector surface, Eq. (34), minimum-curvature spline with tension factor 1 (GMT-routine 'surface') and thin-plate spline radial basis functions below the data points
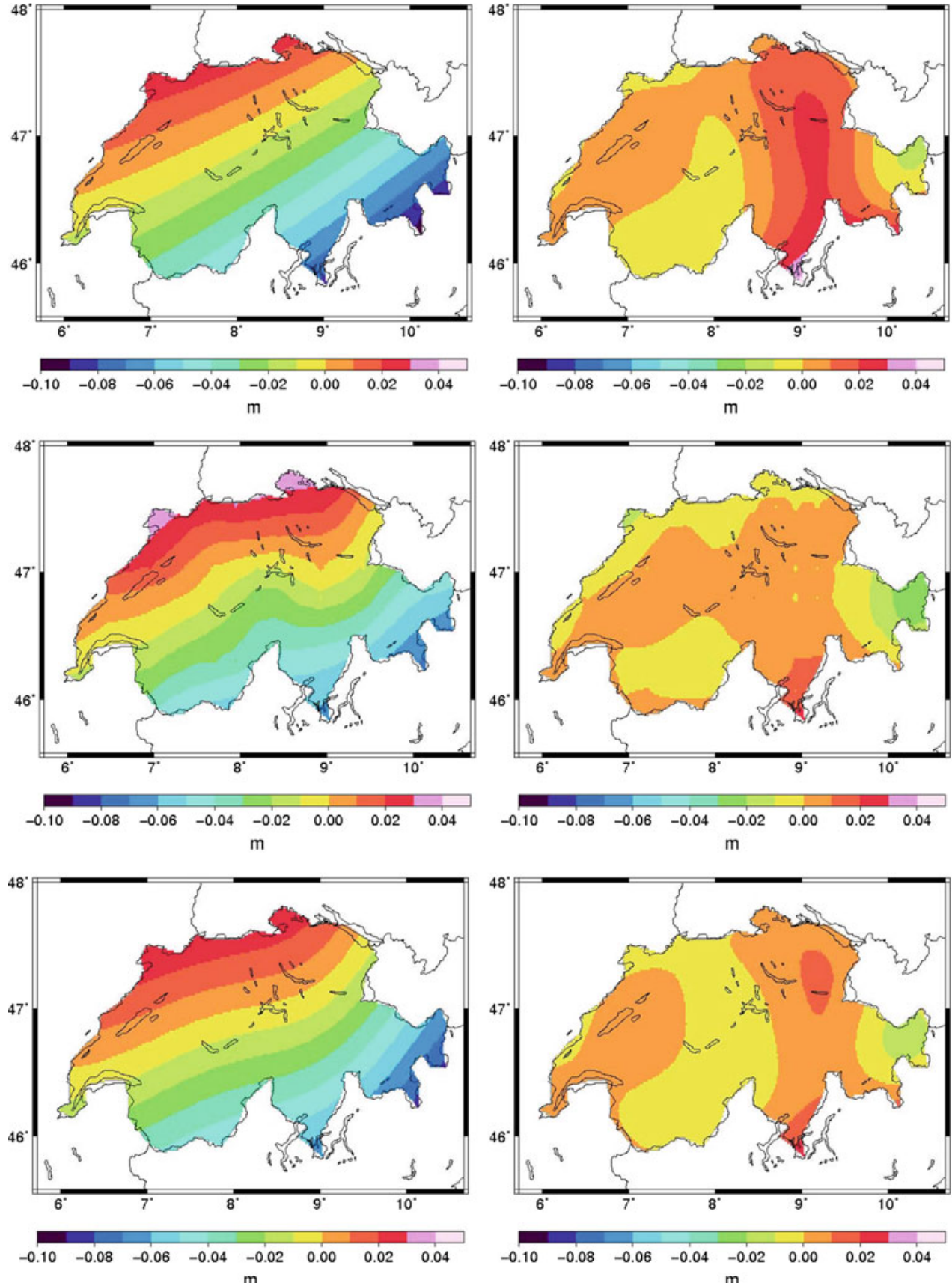

elements $A_{i, m}=f\left(x_{i}, y_{m}\right), \mathbf{p}=\left(p_{1}, \ldots, p_{m}\right)^{T}$ and $\mathbf{q}=$ $\left(q\left(x_{1}\right), \ldots, q\left(x_{J}\right)\right)^{T}$. The regularization parameter $\alpha$ is estimated using GCV. The minimum of the GCV functional is found using Brent's method (Brent 1973). The radial basis functions are placed below the data points, i.e. the number of radial basis functions, $M$, is equal to the number of observed differences between geometric and gravimetric quasi-geoid heights, $J$. The results are shown in Fig. 6 and Table 4. The method performs slightly better than the minimum-curvature spline with tension factor 1 . We also tested other radial basis function locations, e.g. at the nodes of an equiangular grid with $M \approx J$; the maximum differences between these solutions are found to be below $0.1 \mathrm{~cm}$. This is in agreement with (Klees and Wittwer 2007) and (Klees et al. (2008)), who found that the choice of the radial basis function centres does not have a significant effect on the solution.

\section{Comparison with least-squares collocation}

Another popular technique to estimate the differences between gravimetric and geometric quasi-geoid heights is 
Table 4 Statistics of the differences with respect to the reference solution (proposed methodology with full noise covariance matrix) of three correction-surface solutions and two least-squares collocation (LSC) solutions

\begin{tabular}{llllll}
\hline Approach & Min & Max & M & RMS & SD \\
\hline $\begin{array}{l}\text { Minimum-curvature spline } \\
\quad \text { tension factor 1) }\end{array}$ & -2.9 & 2.6 & 0.0 & 0.6 & 0.6 \\
$\begin{array}{l}\text { Thin-plate spline below } \\
\text { data points, Eq. (35) }\end{array}$ & -1.4 & 2.8 & 0.1 & 0.6 & 0.6 \\
$\begin{array}{l}\text { Corrector surface, Eq. (34) } \\
\quad-1.2\end{array}$ & 4.3 & 0.6 & 1.1 & 0.9 \\
$\begin{array}{l}\text { LSC (spherical signal } \\
\quad \text { covariance function) }\end{array}$ & -2.1 & 2.6 & 0.1 & 0.7 & 0.7 \\
$\begin{array}{l}\text { LSC (Matern signal } \\
\quad \text { covariance function) }\end{array}$ & -2.1 & 2.5 & 0.1 & 0.7 & 0.7 \\
\hline
\end{tabular}

The statistics are computed at a 1-min equiangular grid restricted to the target area. Units in cm

least-squares collocation (LSC) (note that least-squares collocation could also be used to directly compute a quasi-geoid using all available data). If these differences have a pronounced spatial trend, as applies to the Swiss data set, we cannot directly use LSC without parameters. A straightforward way of dealing with a trend is to first model the trend as a smoothly varying function of the coordinates, estimate the trend parameters using least-squares and remove the trend from the data. Next, the resulting residuals are treated as realizations of a stationary correlated random variable, the covariance function of that random variable is estimated from the residuals, modelled by a suitably chosen function and then used in LSC without parameters.

The drawback of this approach is that the estimated signal covariance function obtained from the residuals is biased, because it depends in a non-linear way on the estimated trend parameters, which are also erroneous. Moreover, the mean square prediction error at any new station would not be minimum. Therefore, we prefer to use least-squares collocation with parameters and data noise (e.g. Meissl 1976; Tscherning 1978; Moritz 1989), which in this study will be referred to as 'generalized LSC':

$\delta \zeta(x)=\delta \zeta(x, \mathbf{p})+s(x)+n(x)$,

where $\delta \zeta(x)$ is the observed difference between geometric and gravimetric quasi-geoid heights at point $x, \delta \zeta(x, \mathbf{p})$ represents the trend, i.e. the systematic part of the difference, $\mathbf{p}$ is the vector of trend-model parameters, $s(x)$ is a random variable with mean zero and covariance function $C_{s}(x, y)$, which represents the random part of the difference, and $n(x)$ is a random variable with mean zero and covariance function $C_{n}(x, y)$, which represents the noise. The user has to choose the trend model in order to discriminate between the deterministic component of the signal and its random component. This discrimination is to some extent arbitrary, i.e. it is more art than science. However, the choice of the trend model may not be that critical for the following reason: a more complex trend function will lead to a signal covariance function with a shorter correlation length, whereas a simple trend function will lead to a signal covariance function, with a slower decay. If the empirical covariances can be estimated with sufficient accuracy over the relevant range of distances (which must cover the significant range of the signal after trend removal), the solution should not differ much from a solution that uses a more complex trend function. This is the case for the Swiss data set. Therefore, we prefer to use a simple (linear) trend model $\delta \zeta(x, \mathbf{p})=\mathbf{a}(x)^{T} \mathbf{p}$, where $\mathbf{a}(x)$ is a vector of functions, which only depend on $x$. Potential choices are the models discussed in Sect. 5 or even simpler models such as a piecewise constant function or a low-degree algebraic polynomial in ellipsoidal geographic latitude $(\varphi)$ and longitude $(\lambda)$. We used the model (34). Hence,

$\mathbf{a}(x)^{T}=\left(\begin{array}{ll}1 & \cos \varphi \sin \lambda \varphi \lambda \cos \varphi),\end{array}\right.$

and

$\mathbf{p}^{T}=\left(\begin{array}{lll}p_{01} & p_{11} & q_{2}\end{array}\right)$.

A complication from a practical point of view is due to the fact that the trend $h(x, \mathbf{p})$ and the signal covariance function $C_{S}(x, y)$ are not known a priori, but have to be estimated from the data. There are different options for doing this (e.g. Goovaerts 1997). We decided to do this iteratively in the following way:

1. We first estimate the parameters of the trend model using the Gauss-Markov model:

$$
E(\mathbf{q})=\mathbf{A} \mathbf{p}, \quad D(\mathbf{q})=\mathbf{C}_{n},
$$

where the vector $\mathbf{q}$ contains the observations $q\left(x_{j}\right)$, and $\mathbf{A}$ is the design matrix with row vectors $\mathbf{a}\left(x_{j}\right)^{T}$. The least-squares solution is

$$
\hat{\mathbf{p}}_{l s}=\left(\mathbf{A} \mathbf{C}_{n}^{-1} \mathbf{A}\right)^{-1} \mathbf{A}^{T} \mathbf{C}_{n}^{-1} \mathbf{q} .
$$

2. We estimate empirical signal covariances from the residuals $\mathbf{q}-\mathbf{A} \hat{\mathbf{p}}_{l s}$ for a number of lags, and fit a suitable model to this, so obtaining a covariance matrix $\hat{\mathbf{C}}_{s}$ with elements $\hat{C}_{s}\left(x_{i}, x_{j}\right)$. After a visual inspection of the residuals $\mathbf{q}-$ $\mathbf{A} \hat{\mathbf{p}}_{l s}$, we decided to assume isotropy, so estimate isotropic empirical covariances and use an isotropic model covariance function $C_{S}(x, y ; \boldsymbol{\theta})=C_{S}(x-y ; \boldsymbol{\theta})$. The vector of parameters of the model covariance function, $\boldsymbol{\theta}$, is estimated using generalized least-squares, which minimizes

$$
\Phi(\boldsymbol{\theta})=\left(\hat{\mathbf{C}}_{s}-\mathbf{C}_{s}(\boldsymbol{\theta})\right)^{T} \tilde{C}^{-1}\left(\hat{\mathbf{C}}_{s}-\mathbf{C}_{s}(\boldsymbol{\theta})\right),
$$


where $\tilde{C}$ is the covariance matrix of the empirical covariances, $\hat{\mathbf{C}}_{S}$ is the vector of empirical covariances at lags $d_{1}, \ldots, d_{L}, L$ is the number of lags and $\mathbf{C}_{S}(\boldsymbol{\theta})$ is the vector of model covariances at lags $d_{1}, \ldots, d_{L}$. Note that the model covariance function is in general a non-linear function of $\boldsymbol{\theta}$. We follow the same procedure as proposed by Pardo-Iguzquiza and Dowd (2001) and use a GaussNewton algorithm to find the minimizer $\hat{\boldsymbol{\theta}}$ of $\Phi(\boldsymbol{\theta})$. The corresponding covariance function is denoted $C_{S}(x-y ; \hat{\boldsymbol{\theta}})$.

3. We compute an improved trend model $q(x, \hat{\mathbf{p}})$ as

$\hat{\mathbf{p}}=\left(\mathbf{A}^{T} \mathbf{C A}\right)^{-1} \mathbf{A}^{T} \mathbf{C}^{-1} \mathbf{q}, \quad \mathbf{C}=\hat{\mathbf{C}}_{s}+\mathbf{C}_{n}$,

where $\hat{\mathbf{C}}_{S}$ is the signal covariance matrix, which is computed using the model covariance function $C_{S}(x-y ; \hat{\boldsymbol{\theta}})$ of step 2 .

4. We iterate steps 2 and 3 until there are no significant changes in the estimated trend parameters $\hat{\mathbf{p}}$ and the signal covariance function $C_{s}(x-y ; \hat{\boldsymbol{\theta}})$.

5. We predict values $q$ at a point $y$ as

$\hat{q}(y)=q(y, \hat{\mathbf{p}})+\hat{\mathbf{C}}_{s}^{T}(y) \mathbf{C}^{-1}(\mathbf{q}-\mathbf{A} \hat{\mathbf{p}})$,

where $\hat{\mathbf{C}}_{S}(y)$ is the vector of signal covariances with elements $\mathbf{C}_{s}\left(y-x_{j}, \hat{\boldsymbol{\theta}}\right)$.

The empirical covariance function is shown in Fig. 7; 30 lags were estimated; the lag size is about $6 \mathrm{~km}$. The number of pairs per lag varies between 69 and 500 for lags 2 to 30; for lag 1 (distance interval $0-6 \mathrm{~km}$ ) only 13 pairs were available, which leads to a higher uncertainty of the empirical covariance for this lag. This higher uncertainty is taken into account when fitting the covariance model by generalized least-squares. Usually, the lag size is chosen equal to the mean distance between the data points, which for the Swiss data set is about $20 \mathrm{~km}$. We used the smaller lag size to get some ideas about the shape of the covariance function close to the origin. We also used empirical covariance estimates for a lag size of $20 \mathrm{~km}$ using 10 lags, but the estimated model covariance function does not differ significantly from the one computed with a lag size of $6 \mathrm{~km}$. We explain this by the fact that the empirical covariances are weighted when fitting the covariance function model, whereby the weights are chosen proportional to the number of pairs and inverse proportional to the standard deviation of the estimated empirical covariance at that lag.

We tried several model covariance functions, among others a spherical covariance function, a Gaussian covariance function, an exponential covariance function and a Matern covariance function (e.g. Matern 1986; Goovaerts 1997). We assess the goodness of fit ofvarious models using the root mean square error (RMSE). The spherical model is the one

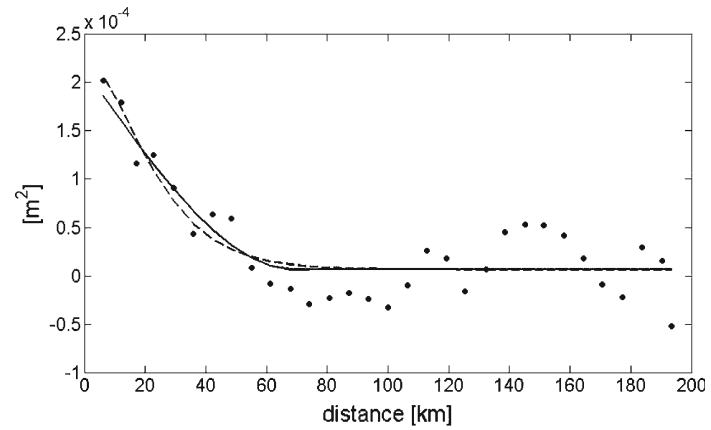

Fig. 7 Empirical (dots) and two model covariance functions used in the least-squares collocation solution: Matern covariance function (solid line) and spherical covariance function (dashed line)

Table 5 Estimated parameters for two model signal covariance functions, which are used in generalized LSC

\begin{tabular}{lllll}
\hline & $C_{0}\left(\mathrm{~m}^{2}\right)$ & $C_{1}\left(\mathrm{~m}^{2}\right)$ & $C_{2}(\mathrm{~km})$ & $C_{3}(-)$ \\
\hline $\begin{array}{l}\text { Spherical } \\
\text { covariance function }\end{array}$ & $2.773 \times 10^{-4}$ & $2.077 \times 10^{-4}$ & 65.2 & \\
$\begin{array}{l}\text { Matern } \\
\text { covariance function }\end{array}$ & $2.676 \times 10^{-4}$ & $2.170 \times 10^{-4}$ & 10.1 & 1.99 \\
\hline
\end{tabular}

with the smallest RMSE $\left(2.33 \times 10^{-5}\right)$, though the RMSE of the Matern model is very close to it $\left(3.46 \times 10^{-5}\right)$; these two models are given as

$C_{S}(d)=\left\{\begin{array}{ll}\sigma^{2}-C_{0}-C_{1}\left[\frac{3}{2} \frac{d}{C_{2}}-\frac{1}{2}\left(\frac{d}{C_{2}}\right)^{3}\right] & \text { for } d<C_{2} \\ \sigma^{2}-\left(C_{0}-C_{1}\right) & \text { for } d \geq C_{2} \\ \sigma^{2} & \text { for } d=0\end{array}\right.$,

and

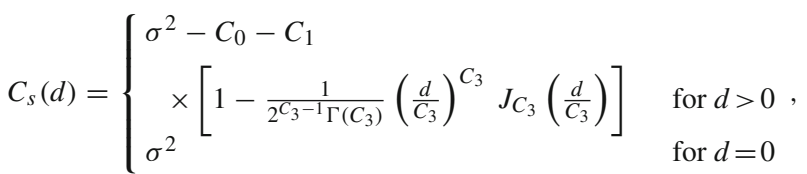

respectively. $d$ is the distance between two points, $\sigma^{2}$ is the signal variance (i.e. the variance of the residuals $\mathbf{q}-A \hat{\mathbf{p}}$, which after the final iteration is $4.9 \times 10^{-4} \mathrm{~m}^{2}$ ), $\Gamma$ is the Gamma-function and $J_{S}$ is the modified Bessel function of the second kind of order $s$. Figure 7 shows the fitted spherical and Matern covariance functions and Table 5 contains the final parameters for each of the two models.

The fitted spherical and Matern signal covariance functions provide generalized LSC solutions, which are very close to each other; the maximum difference is $0.4 \mathrm{~cm}$ and the RMS difference is $0.1 \mathrm{~cm}$. Figure 8 shows the spatial pattern of the solution using the Matern covariance function and the difference with respect to the reference solution 
Fig. 8 Least-squares collocation solution (Matern signal covariance function) and differences with respect to the reference solution (proposed methodology with full noise covariance matrix)
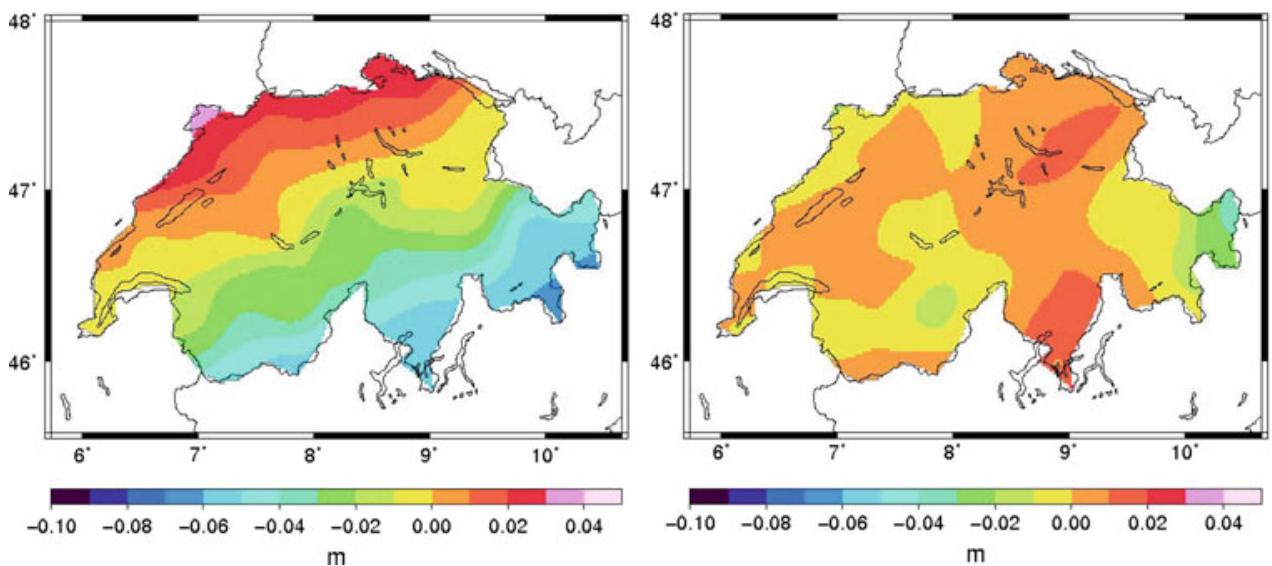

(innovation function with $d=120 \mathrm{~km}$ and full noise covariance matrix). Table 4 contains the statistics of the differences. Note that the amplitude of the trend component is much larger than the amplitude of the random component: at the GNSSlevelling points, the trend component varies between -7.3 and $2.8 \mathrm{~cm}$, whereas the random component varies between -1.2 and $1.3 \mathrm{~cm}$.

The a posteriori prediction errors (trend and random part) at the GNSS-levelling points take up values between \pm 0.9 and $\pm 2.1 \mathrm{~cm}$. About $70 \%$ comes from the random part, and the remaining $30 \%$ comes from the deterministic part. We also computed the a posteriori prediction errors (trend and random part) at 1000 points randomly distributed over the target area; they vary between \pm 2.0 and $\pm 3.1 \mathrm{~cm}$.

\section{Discussion}

\subsection{The proposed methodology}

The proposed methodology is one of several strategies to combine a gravimetric quasi-geoid model with GNSSlevelling data. The assumption that underlies this approach is that (1) the lack of high-quality and dense gravity data outside the target area is the main contributor to the observed differences between gravimetric and geometric quasi-geoid heights, (2) the quality of quasi-geoid heights derived from GNSS-levelling data is at least comparable to the quality of the gravimetric quasi-geoid and (3) the density of the GNSSlevelling points is enough to capture most of the signal in the observed differences (Table 6).

Assumption (1) is not valid if systematic, long-wavelength errors are present in geometric quasi-geoid heights, which, unfortunately, is a quite common situation in practice. For example, long-wavelength errors in levelling networks propagate into levelling-based heights and, therefore, also in the observed differences between geometric and gravimetric quasi-geoid heights. Then, the problems (2) and (6) are not the correct model to combine a gravimetric quasi-geoid with GNSS-levelling data. The practical implication of this is that the innovation function, Eq. (16), cannot be interpreted anymore as a correction to the gravimetric (quasi-) geoid in order to obtain an improved (quasi-) geoid, which is compatible with gravity anomalies and GNSS-levelling data. Without additional information, it is not possible to distinguish between long-wavelength errors in the gravimetric (quasi-) geoid caused by, e.g. the lack of suitable gravity data inside or outside the target area and long-wavelength systematic errors in levelling-based heights caused by deformations of the levelling network. Then, the corrector-surface approach may be a valid choice to deal with these long-wavelength errors provided that it is only used to facilitate the transformation of ellipsoidal heights into levelling-based heights. The question is whether the methodology developed in Sect. 2 can also be used for that purpose. Our answer is 'yes it can'. In order to understand this, we may have a look at Eq. (17), which represents the solution to problem (6) after discretization of the integral equations. Equation (17) can formally be written as

$u(x)=\sum_{i=1}^{I} \chi_{i} \Psi\left(x, y_{i}\right)$

where $\Psi\left(x, y_{i}\right)$ is a radial basis function with centre $y_{i}$, i.e. the solution $u(x)$ to problem (6) is written as a linear combination of radial basis functions. Equations $(23,24)$ is the (regularized) least-squares approximation of the vector of unknown coefficients $\chi=\left(\chi_{i}\right)$. Hence, solution (25) can formally be interpreted as a least-squares radial basis function approximation to the differences between geometric and gravimetric height anomalies. In the presence of systematic errors of non-gravitational origin in the differences between geometric and gravimetric (quasi-) geoid heights, the proposed methodology provides a corrector surface, which can be used like any other corrector surface to facilitate the transformation of ellipsoidal heights into levelling-based heights. The only difference with respect to more traditional corrector 
Table 6 Statistics of the residuals at the GNSS-levelling points for various methods

Units in $\mathrm{cm}$. The last column shows the effective regularization parameter $\alpha_{\mathrm{gcv}, \text { eff }}$ used to compute the solution

\begin{tabular}{lrrrrl}
\hline & Min & Max & Mean & RMS & $\begin{array}{l}\text { Effective } \\
\text { regularization } \\
\text { parameter }\end{array}$ \\
\hline $\begin{array}{l}\text { Proposed methodology; full noise covariance matrix } \\
\quad \text { (reference solution) }\end{array}$ & -4.8 & 5.3 & -0.2 & 2.0 & 0.0619 \\
Proposed methodology; diagonal noise covariance matrix & -3.9 & 5.3 & 0.0 & 1.8 & 0.0019 \\
Proposed methodology; unit noise covariance matrix & -3.7 & 4.9 & 0.0 & 1.8 & 0.0017 \\
Prutkin and Klees (2008); gridded data & -4.2 & 5.3 & -0.1 & 1.9 & 0.1219 \\
Prutkin and Klees (2008); original data & -3.8 & 5.1 & 0.0 & 1.8 & 0.6101 \\
Corrector surface, Eq. (34) & -5.0 & 6.9 & 0.5 & 2.2 & 0.0000 \\
Thin-plate spline below data points, Eq. (35) & -5.6 & 5.7 & 0.0 & 2.1 & 0.0199 \\
LSC (spherical signal covariance function) & -4.6 & 5.5 & 0.0 & 1.8 & 0.0000 \\
LSC (Matern signal covariance function) & -4.7 & 5.6 & 0.0 & 1.9 & 0.0000 \\
\hline
\end{tabular}

surface models like those of Eq. (33) is the choice of the basis functions.

Assumption (2) will only be met if the occupation times of the benchmarks with GNSS are sufficiently long and if the data processing is done carefully. For instance, in the Netherlands, the majority of the benchmarks has been occupied with GNSS for 1-2 hours, which is not enough to determine the ellipsoidal height with an accuracy of 1-2 cm.

Assumption (3) requires enough GNSS-levelling points within the target area. Only half-wavelengths larger than the mean distance between GNSS-levelling points can be captured. For the Swiss data set, this limits to wavelengths larger than about $20 \mathrm{~km}$. Smaller features are left unmodelled.

\subsection{The numerical experiment}

The experiments with the Swiss data set have shown that taking noise covariances into account is important for precise quasi-geoid modelling. We explain this by the fact that the noise in differences between geometric and gravimetric quasi-geoid heights at the GNSS-levelling points is highly correlated. The main contributors are the noise correlations among normal heights and among gravimetric quasi-geoid heights. The maximum error of $1.1 \mathrm{~cm}$ that is introduced if noise correlations are neglected does not seem to be much, in particular relative to the precision of the quasi-geoid, which is of the order of $\pm 2 \mathrm{~cm}$. However, one should remember that for the Swiss data set, the maximum difference between geometric and gravimetric quasi-geoid heights is only $9.9 \mathrm{~cm}$. Hence, neglecting noise correlations introduces errors up of $10 \%$ of the maximum signal! As the errors scale with the magnitude of the signal, larger errors are expected if the differences between geometric and gravimetric quasi-geoid heights increase. Therefore, we recommend to put effort into getting access to full noise covariance information for
GNSS ellipsoidal heights, levelling-based heights and gravimetric (quasi-) geoid heights, when computing an optimal (quasi-) geoid from gravity anomalies and GNSS-levelling data. The individual covariance matrices must refer to the same datum, which in practice may require the application of S-transformations (Baarda 1981) before the three noise covariance matrices are added to get the noise covariance matrix of the differences between geometric and gravimetric (quasi-) geoid heights (cf. Eq. (30)). Moreover, it should be investigated whether cross correlations between noise in levelling-based heights and gravimetric (quasi-) geoid heights need to be taken into account, because both may rely upon the same gravity data set. Another aspect which needs further investigation is the contribution to $\mathbf{Q}_{\zeta}$ (the noise covariance matrix of the gravimetric quasi-geoid heights, see Eq. (30)) of commission and omission errors of the global reference gravity model. This information was not available for the Swiss data set. Hence, the variance factor $\sigma_{\zeta}^{2}$, Eq. (32), is too optimistic.

\subsection{The corrector-surface approach}

The main advantage of the proposed methodology compared with the corrector-surface approach is that (1) no subjective choice of a parametric model is needed and (2) the combined (quasi-) geoid solution is compatible with all data (i.e. with the gravity anomaly data set and with the GNSS-levelling data set), whereas the corrector-surface approach yields corrections that are not compatible with the gravity anomaly data set. However, the proposed methodology is also not free of some choices to be made: (1) the choice of the depths of the sources and (2) the choice of the regularization parameter. Both determine the smoothness of the solution. It is difficult to decide which pair of depth/regularization parameter is the correct one without knowing the exact quasi-geoid. In this 
study, we used the available information about the standard deviations of the geometric quasi-geoid heights at the benchmarks as indicator of over- or underfit. This information was complemented by a visual inspection of the innovation function. Local gradients around data points were interpreted as an indicator of overfit. Very smooth innovation functions were considered as the result of underfit. When applying these subjective criteria, different pairs of depth and regularization parameter may be admissible choices. However, from many numerical experiments with the Swiss data set, we found that different pairs of admissible parameters yield innovation functions, which are identical within a few millimetres.

The corrector-surface approach may only offer an alternative to the proposed approach if the differences between geometric and gravimetric quasi-geoid heights are very smooth. This depends on the accuracy and density of the gravity anomalies inside and outside the target area. For instance, if little data are available outside the target area, a more complicated pattern of corrections is expected if the gravimetric (quasi-) geoid is combined with GNSS-levelling data. The same applies if the quality of gravity data outside the target area is superior to the quality of gravity data inside the target area, a situation that is not unusual in practice. Then, it may be a non-trivial task to find a suitable analytical model for the corrector surface, which is able to follow this more complicated spatial pattern sufficiently well. If the user wants to apply the corrector-surface approach, we recommend to follow the approach of van Loon (2008), which comprises outlier detection or robust estimation techniques, VCE to properly scale the noise covariance matrices, and significance tests of the estimated model parameters. Outlier detection may be a problem in practice, in particular if the number of GNSS-levelling points is low or if there are isolated GNSS-levelling points. An example are the two most eastern GNSS-levelling points of the Swiss data set, which are poorly controled by other GNSS-levelling points.

There may be better alternatives to the popular corrector surfaces, Eq. (33). For instance, for the Swiss data set better results were obtained with a minimum-curvature (harmonic) spline (Smith and Wessel 1990), which is implemented e.g. in the 'surface' routine of the GMT software package (Wessel and Smith 1991) or with least-squares radial basis function approximations. If the latter are being used, we recommend to choose the GNSS-levelling data points as radial basis function centres for simplicity reasons and to estimate the regularization parameter using GCV. No matter what corrector surface model is used, the corrector-surface approach does not take into account that the differences between geometric and gravimetric (quasi-) geoid heights multiplied by the normal gravity (which can be considered as a constant scaling factor without introducing significant errors) may be the trace of a harmonic function on the Earth's surface. Then, they provide solutions which are not compatible anymore with the gravity anomaly data set.

\subsection{Least-squares collocation}

Least-squares collocation applied to the differences between geometric and gravimetric quasi-geoid heights is another alternative to the methodology developed in Sect. 2. As the differences usually show a significant trend and the data are noisy, generalized LSC (i.e. LSC with parameters and data noise) should be used. The inherent problem then is that the trend function is not known a priori, but has to be estimated from the data together with the parameters of the signal covariance function. The iterative approach presented in this paper is a simple solution to this problem; some alternatives are known in geostatistics (e.g. Goovaerts 1997), but have not been tested in this study. The approach of van Loon (2008) may be used to find a good trend model within the class of linear models. Another, though more theoretical, problem is that the signal covariance function of the differences between geometric and gravimetric quasi-geoid heights is in general not compatible with the signal covariance function of the gravity anomalies. Another choice to be made in generalized LSC is the type of analytical covariance function. We recommend to fit various analytical covariance functions to the empirical covariances and to choose the one that is the optimal one according to some statistical measure. In this study, we used the root mean square error (RMSE) as a measure of optimality. Many other measures are known in geostatistical literature (e.g. Isaaks and Srivastava 1990; Goovaerts 1997; Webster and Olivier 2007) such as Akaike's information criterion AIC (Akaike 1973). For the Swiss data set and a simple linear algebraic polynomial in latitude and longitude as trend model, the spherical covariance function and the Matern covariance function had the smallest RMSE (and AIC) and in this sense performed better than other covariance models. Predictions made with both models differ only by a few millimetres, though the models behave differently for short distances.

A better methodology than generalized LSC applied to the differences between geometric and gravimetric (quasi-) geoid heights would be generalized LSC applied to gravity anomalies and GNSS-levelling data. In this case, the signal covariance function of the disturbing potential and the crosscovariance function between gravity anomalies and disturbing potentials are derived from the signal covariance function of gravity anomalies by covariance propagation. Then, there is no compatibility problem anymore as no signal covariance function of the differences between geometric and gravimetric quasi-geoid heights needs to be estimated. This, however, requires the access to all original data. Nevertheless, it would be interesting to compare such an approach with generalized LSC applied to differences between gravimetric and 
geometric (quasi-) geoid heights. A practical advantage of the latter approach is that new corrections to the gravimetric (quasi-) geoid heights can easily be computed once new GNSS measurements at benchmarks become available. The more strict approach of processing all available data together would require a completely new computation of the (quasi-) geoid. Moreover, it is likely that GNSS-levelling data will not contribute much to the combined solution as long as their number is much smaller than the number of gravity anomalies.

\section{Summary and conclusion}

Local quasi-geoid modelling from gravity anomalies and GNSS-levelling data can be formulated as a Cauchy boundary value problem for the Laplace equation. The deterministic approach by Prutkin and Klees (2008), which solves this problem approximately in the vicinity of the target area, has been extended to noisy data. The method was applied to a real data set for Switzerland. The effect of noise correlations and spatially varying noise variances on the combined quasi-geoid solution were quantified, and the method was compared with the corrector-surface approach using various corrector surface models and with generalized LSC. The main conclusion is that noise correlations need to be taken into account for precise quasi-geoid modelling. Otherwise, errors of about $10 \%$ of the differences between geometric and gravimetric quasi-geoid heights can be introduced easily. The largest errors occur in areas with little GNSS-levelling data or in the neighborhood of isolated GNSS-levelling points. Compared with the popular corrector-surface approach, the proposed method has the advantage that no choice of a parametric model needs to be done and that the combined quasigeoid solution is consistent with both data sets. Compared with generalized LSC applied to differences between geometric and gravimetric quasi-geoid heights at benchmarks, the proposed method is less complex and numerically easier to implement. Moreover, generalized LSC also requires the choice of a parameteric trend model, though the solution may be less sensitive to this choice than any corrector surface solution.

Acknowledgments Urs Marti from the Federal Office of Topography Swisstopo provided the test data sets. Software written by Jasper van Loon has been used to compute some corrector surface models. This support is gratefully acknowledged. We also acknowledge the detailed comments of three anonymous reviewers and of the editor, which helped us improving the quality of the manuscript.

Open Access This article is distributed under the terms of the Creative Commons Attribution Noncommercial License which permits any noncommercial use, distribution, and reproduction in any medium, provided the original author(s) and source are credited.

\section{References}

Akaike H (1973) Information theory and an extension of the maximum likelihood principle. In: Petrov BN, Csáki F (eds) Second international symposium on information theory. Akadémiai Kiadó, Budapest, pp 267-281

Baarda W (1981) S-transformations and criterion matrices; Publications on Geodesy 18 (vol 5 no 1), second revised edition. Delft, The Netherlands

Brent RP (1973) Algorithms for minimization without derivatives. Prentice-Hall, Englewood Cliffs

Craven P, Wahba G (1979) Smoothing noisy data with spline functions. Numer Math 31:377-403

de Bruijne AJT, Haagmans RHN, de Min EJ (1997) A preliminary North Sea Geoid model GEONZ97. Rep MDGAP-9735, Directoraat-Generaal Rijkswaterstaat, Meetkundige Dienst, Delft, The Netherlands

de Min E (1996) De geoide voor Nederland. Nederlandse Commissie voor Geodesie, Groene Serie, vol 34. Delft, The Netherlands

Denker H, Torge W (1998) The European gravimetric quasigeoid EGG97-An IAG supported continental enterprise. In: Forsberg R, Feissel M, Dietrich R (eds) Geodesy on the Move-Gravity, Geoid, Geodynamics and Antarctica. IAG Symp Vol 119: 249-254. Springer, Berlin

Featherstone WE (1998) Do we need a gravimetric geoid or a model of the base of the Australian height datum to transform GNSS heights? Aust Surv 43:273-280

Featherstone WE (2000) Refinement of a gravimetric geoid using GNSS and levelling data. J Surv Eng 126:27-56

Fotopoulos G (2005) Calibration of geoid error models via a combined adjustment of ellipsoidal, orthometric and gravimetric geoid height data. J Geod 79:111-123

Goovaerts P(1997) Gostatistics for natural resources evaluation. Oxford University Press, New York

Grebenitcharsky RS, Rangelova EV, Sideris MG (2005) Transformation between gravimetric and GNSS/levelling-derived geoids using additional gravity information. J Geodyn 39:527-544

Heck B (1997) Formulation and linearization of boundary value problems: from observables to a mathematical model. In: Sanso F, Rummel R (eds) Geodetic boundary value problems in view of the one centimeter geoid. Lecture notes in earth sciences. Springer, Berlin pp 121-160

Heiskanen WA, Moritz H (1967) Physical geodesy. Freeman, San Francisco

Isaaks EH, Srivastava RM (1990) An introduction to applied geostatistics. Oxford University Press, USA

Kellogg OD (1929) Foundations of potential theory. Springer, Berlin

Klees R, Wittwer T (2007) A data-adaptive design of a spherical basis function network for gravity field modelling. In: Tregoning P, Rizos C (eds) Dynamic planet-monitoring and understanding a dynamic planet with geodetic and oceanographic tools, International Association of Geodesy Symposia, vol 130. Springer, Berlin, pp 323-328

Klees R, Tenzer R, Prutkin I, Wittwer T (2008) A data-driven approach to local gravity field modelling using spherical radial basis functions. J Geod 82:457-471

Matern B (1986) Spatial variation. Lecture notes in statistics, 2nd edn, vol 36. Springer, New York

Meissl P (1971) Preparations for the numerical evaluation of secondorder Molodensky-type formulas. Report 163, Dept Geod Sci \& Surv, Ohio State University, Columbus

Meissl P (1976) Hilbert spaces and their application to geodetic leastsquares problems. Boll Geod Sci Affini 35:49-80 
Molodensky MS, Eremeev VF, Yurkina MI (1962) Methods for study of the external gravitational field and figure of the earth. Israeli Programme for the Translation of Scientific Publications, Jerusalem

Moritz H (1989) Advanced physical geodesy, 2nd edn. Wichmann, Karlsruhe

Nahavandchi N, Soltanpour A (2006) Improved determination of heights using a conversion surface by combining gravimetric quasi-geoid /geoid and GNSS-levelling height differences. Stud Geophys Geod 50:165-180

Pardo-Iguzquiza E, Dowd PA (2001) VARIOG2D: a computer program for estimating the semi-variogram and its uncertainty. Comput Geosci 27:549-561

Pavlis NK, Holmes SA, Kenyon SC, Factor JK (2008) An Earth gravitational model to degree 2160: EGM2008. Paper presented at the EGU General Assembly 2008, Vienna, Austria

Prutkin I, Klees R (2008) On the non-uniqueness of local quasi-geoids computed from terrestrial gravity anomalies. J Geod 82:147-156
Smith WHF, Wessel P (1990) Gridding with continuous curvature splines in tension. Geophysics 55:293-305

Tscherning CC (1978) Introduction to functional analysis with a view to its application in approximation theory. In: Moritz H, Sünkel H (eds) Approximation methods in geodesy. Wichmann H, Karlsruhe pp 157-192

van Loon J (2008) Functional and stochastic modelling of satellite gravity data, 231 p. PhD thesis, Delft University of Technology, Delft, The Netherlands

Webster R, Olivier MA (2007) Geostatistics for environmental scientists, 2nd edn. Wiley, New York

Wessel P, Smith WHF (1991) Free software helps map an display data. EOS Trans AGU 72:441

Wong L, Gore R (1969) Accuracy of geoid heights from modified Stokes kernels. Geophys J R Astron Soc 18:81-91 